\title{
Effects of long-term exercise on plasma adipokine levels and inflammation-related gene expression in subcutaneous adipose tissue in sedentary dysglycaemic, overweight men and sedentary normoglycaemic men of healthy weight
}

\author{
Sindre Lee ${ }^{1,2} \cdot$ Frode Norheim $^{1} \cdot$ Torgrim M. Langleite $^{1} \cdot$ Hanne L. Gulseth ${ }^{2,3} \cdot$ Kåre I. Birkeland $^{2,4}$. \\ Christian A. Drevon ${ }^{1}$
}

Received: 11 January 2019 / Accepted: 1 March 2019 / Published online: 22 April 2019

(C) Springer-Verlag GmbH Germany, part of Springer Nature 2019, corrected publication 2022

\begin{abstract}
Aims/hypothesis Obesity and insulin resistance may be associated with altered expression and secretion of adipokines. Physical activity can markedly improve insulin sensitivity, but the association with adipokines remains largely unknown. In this study, we examined the effects of physical activity on the subcutaneous white adipose tissue (scWAT) secretome and its relationship to insulin sensitivity.

Methods As reported previously, we enrolled 26 sedentary, middle-aged men (13 dysglycaemic and overweight; 13 normoglycaemic and of healthy weight) into a 12 week, supervised, intensive physical exercise intervention that included two endurance and two resistance sessions each week. Insulin sensitivity was measured as the glucose infusion rate from a euglycaemic-hyperinsulinaemic clamp. In our previous study, we measured maximum oxygen uptake, upper- and lower-body strength and a range of circulating biomarkers, and quantified adipose tissue depots using MRI and magnetic resonance spectroscopy. We have now performed global mRNA sequencing, microarrays and RT-PCR of scWAT and skeletal muscle biopsies, and quantified selected plasma adipokines by ELISA.

Results Insulin sensitivity increased similarly in both dysglycaemic (45\%) and normoglycaemic (38\%) men after 12 weeks of exercise, as reported previously. mRNA sequencing of scWAT revealed 90 transcripts that responded to exercise in dysglycaemic men, whereas only marginal changes were observed in normoglycaemic men. These results were validated using microarrays and RT-PCR. A total of 62 out of 90 transcripts encoded secreted proteins. Overall, 17 transcripts were upregulated and 73 transcripts were downregulated. Downregulated transcripts included several macrophage markers, and were associated with inflammatory and immune-related pathways. Levels of these immune-related transcripts were enhanced in dysglycaemic men vs normoglycaemic men at baseline, but were normalised after the exercise intervention. Principal component and correlation analyses revealed inverse correlations between levels of these immune-related transcripts and insulin sensitivity at baseline, after the intervention, and for the change between baseline and after the intervention. In addition, levels of these transcripts at baseline could predict exercise-induced improvements in insulin sensitivity. Adipokine levels in scWAT (but not in skeletal muscle) were significantly correlated with corresponding plasma adipokine concentrations, as exemplified by leptin, high-molecular-weight adiponectin and secreted frizzledrelated protein 4 (SFRP4). SFRP4 mRNA was the most exercise-responsive transcript in scWAT from dysglycaemic men, and plasma SFRP4 concentrations were reduced in dysglycaemic men, but not in normoglycaemic men, after 12 weeks of exercise.
\end{abstract}

Electronic supplementary material The online version of this article (https://doi.org/10.1007/s00125-019-4866-5) contains peer-reviewed but unedited supplementary material, which is available to authorised users.

Sindre Lee

sindre.lee@medisin.uio.no

1 Department of Nutrition, Institute of Basic Medical Sciences, Faculty of Medicine, University of Oslo, Sognsvannsveien 9, 0372 Oslo, Norway
2 Department of Endocrinology, Morbid Obesity and Preventive Medicine, Oslo University Hospital, Oslo, Norway

3 Department of Chronic Diseases and Ageing, Norwegian Institute of Public Health, Oslo, Norway

4 Institute of Clinical Medicine, Faculty of Medicine, University of Oslo, Oslo, Norway 


\section{Research in context}

\section{What is already known about this subject?}

- Studies have indicated that lifestyle interventions have beneficial effects on adipokines and insulin resistance, but have only focused on a few adipokines that are mostly related to visceral adipose tissue and weight loss

- Long-term physical exercise has profound effects on the subcutaneous white adipose tissue (scWAT) secretome and insulin sensitivity in mice, but studies in humans are lacking

\section{What is the key question?}

- How does 12 weeks of physical exercise influence global expression of secreted factors in scWAT and plasma concentrations of such factors in overweight men with dysglycaemia compared with healthy weight men with normoglycaemia?

\section{What are the new findings?}

- Using global mRNA sequencing, we discovered a distinct effect of 12 weeks of exercise on the scWAT secretome specifically for dysglycaemic men, with minimal alterations observed in normoglycaemic men

- These secreted factors were associated with inflammation, and correlated negatively with insulin sensitivity

- Alterations in mRNA expression in scWAT were mirrored in plasma adipokine concentrations, as exemplified by SFRP4

How might this impact on clinical practice in the foreseeable future?

- Our data may aid the discovery of plasma biomarkers of insulin resistance

Conclusions/interpretation This study indicates that scWAT may be an important mediator of exercise-induced improvements in insulin sensitivity, especially in overweight dysglycaemic individuals at increased risk of developing type 2 diabetes.

Keywords Adipokines $\cdot$ Humans $\cdot$ Insulin sensitivity $\cdot$ Long-term exercise $\cdot$ Prediabetes $\cdot$ Subcutaneous adipose tissue

$\begin{array}{ll}\text { Abbreviations } \\ \text { scWAT } & \begin{array}{l}\text { Subcutaneous white adipose } \\ \text { tissue }\end{array} \\ \text { GIR } & \begin{array}{l}\text { Glucose infusion rate } \\ \text { MRS }\end{array} \\ \text { Magnetic resonance spectroscopy } \\ \text { SFRP4 } & \text { Secreted frizzled-related protein } 4 \\ \dot{V} \mathrm{O}_{2 \max } & \text { Maximal oxygen uptake }\end{array}$

\section{Introduction}

Adipose tissue is an active endocrine organ that expresses and secretes multiple metabolically active factors such as leptin, adiponectin, IL-6 and TNF- $\alpha$ [1-3]. These secreted factors are involved in metabolic and inflammatory processes and may act in a paracrine or endocrine way, altering metabolism in the liver, pancreas, skeletal muscle and central nervous system [3, 4]. Dysregulation of these signal molecules is closely related to adipocyte hypertrophy and insulin resistance [5-7], and has been characterised in several studies [3,8-10].

Insulin resistance is a hallmark of type 2 diabetes mellitus and is closely linked to lifestyle variables such as diet and physical activity [11-14]. Physical activity can substantially increase insulin sensitivity, and exercise-induced alterations in subcutaneous white adipose tissue (scWAT) may affect wholebody metabolic health $[4,15,16]$. Mediators of these effects may involve extensive adaptations in adipokine expression [4, 16]. Numerous studies have focused on the effect of different types of exercise on circulating levels of adipose tissuederived factors [3, 17-19]. However, the main body of literature on adipokines and exercise is limited to plasma analyses of one or a few targets and the effects of acute exercise [3, 19-22] confounded by weight loss [23] or focused on only visceral adipose tissue $[3,17,18]$. Few studies have addressed long-term exercise-induced alterations in scWAT expression of such factors $[22,24]$, and these studies have only been performed in insulin-sensitive women [22, 24]. In addition, whereas skeletal muscle has been extensively studied as regards physical exercise [25], significantly less focus has been placed on adipose tissue in this aspect.

In the present study, we investigated the effect of long-term physical exercise on scWAT transcript levels and potential links to insulin sensitivity. We performed global mRNA sequencing on biopsies, together with ELISA measurements of selected 
plasma adipokines, and quantified insulin sensitivity using a euglycaemic-hyperinsulinaemic clamp. We hypothesised that 12 weeks of combined endurance and strength training would promote distinct alterations in the scWAT transcriptome among overweight men with dysglycaemia and men of healthy weight with normoglycaemia, and that these changes would correlate with insulin sensitivity.

\section{Methods}

\section{Participants and experimental methods standardisation}

The MyoGlu study was a controlled clinical exercise intervention trial (ClinicalTrials.gov registration no. NCT01803568) in 26 sedentary white ( $<1$ exercise session/week) men aged 40-65 years of Scandinavian origin performed in 2011-2012 in Oslo, Norway (Fig. 1) [26]. All participants gave written informed consent, and the study was approved by the
Regional Committee for Medical and Health Research Ethics North (Tromsø, Norway; ref. no. 2011/882) and adhered to the Declaration of Helsinki. The study was designed to in-depth phenotypically characterise the effects of an intensive exercise intervention across the glucometabolic spectrum.

We included 13 dysglycaemic, overweight men (fasting glucose $\geq 5.6 \mathrm{mmol} / 1,2 \mathrm{~h}$ OGTT $\geq 7.8 \mathrm{mmol} / 1$ or insulin resistance [HOMA-IR >2.0]; BMI 26.8-32.5 kg/m ${ }^{2}$ ) and 13 normoglycaemic men of healthy weight (fasting glucose $<5.6 \mathrm{mmol} / \mathrm{l}$ and $2 \mathrm{~h} \mathrm{OGTT}<7.8 \mathrm{mmol} / \mathrm{l}$ and without a family history of diabetes; BMI 20.9-26.7 kg/m²). Exclusion criteria included smoking, a family history of diabetes (for control participants only), known hypertension, liver or kidney disease, chronic inflammatory diseases or use of a medication expected to affect glucose metabolism (e.g. lipid-lowering or antihypertensive medications, acetylsalicylic acid, corticosteroids).

Before the intervention, the participants refrained from physical exercise and alcohol for 2 days before testing (Fig. 1). The last session of the 12 week intervention consisted of an endurance session with intervals performed at $85 \%$ of

a Enrolled subjects

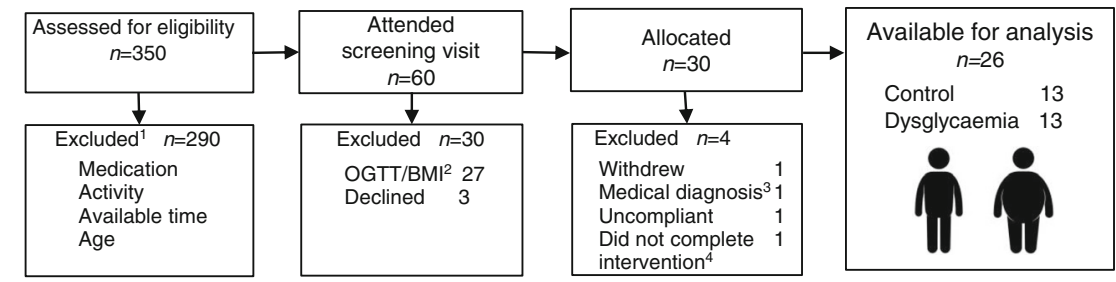

b Timeline

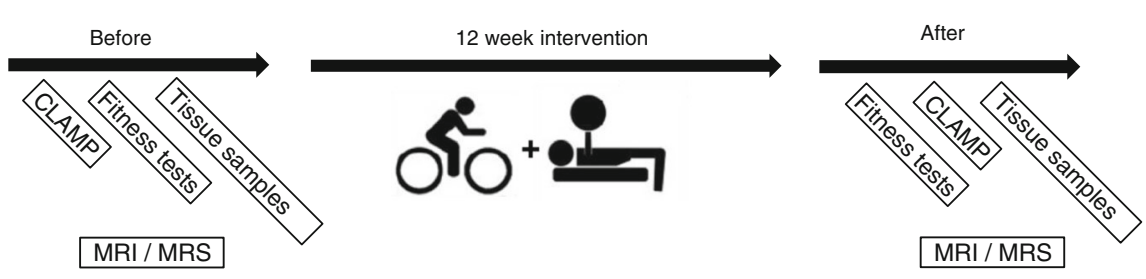

C Exercise sessions each week

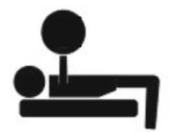

Strength session 1 $10 \mathrm{~min}$ aerobic wam session 1 three sets of Intervals at $>90 \%$ of maximum chest press, cable pull-down, shoulder press, seated rowing abdominal crunch, back extension

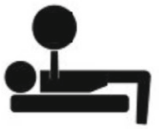

Strength session 2 Same as session 1

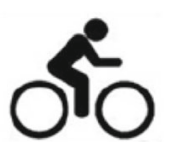

Endurance session 2:

Intervals at $85 \%$ of maximum heart rate with 2 min light-load rests
Fig. 1 Study design. (a) A total of 26 sedentary men (13 of healthy weight with normoglycaemia [control] and 13 overweight with dysglycaemia) were recruited into two groups. ${ }^{1}$ No participants were excluded because of lack of available time; a very small number were excluded because they did not meet the age criterion, $\sim 70 \%$ were excluded because of medication or because of family members with diabetes (controls); $\sim 30 \%$ were excluded because they had been too active in the past year. ${ }^{2}$ OGTT response did not match phenotype, e.g. an obese person had a normal OGTT response. ${ }^{3}$ One participant was diagnosed with acute coronary syndrome. ${ }^{4}$ Due to illness. (b) The participants donated tissue samples and underwent several tests at baseline, including a euglycaemic-hyperinsulinaemic clamp, before undertaking 12 weeks of an intensive physical exercise intervention followed by re-testing. Three days passed between the last bout of exercise and the euglycaemichyperinsulinaemic clamp after the intervention. (c) The intervention consisted of four exercise sessions each week 
maximum heart rate 3 days prior to repeated testing (Fig. 1). Maximal oxygen uptake $\left(\dot{V} \mathrm{O}_{2 \max }\right)$ tests and maximum strength tests were performed several days ( $>3$ days) before the euglycaemic-hyperinsulinaemic clamp and tissue sampling (Fig. 1). Tests were performed under similar conditions on separate days both before and after the intervention, with some exceptions for $\mathrm{MRI} /$ magnetic resonance spectroscopy (MRS) due to scanner availability.

\section{Diet}

The habitual diet of each participant was recorded using a validated food frequency questionnaire $[27,28]$. Calculations were performed using the food database AE-10 and the KBS v.7.1 (Kostberegningssystem, Oslo, Norway) food and nutrients calculation system. Alcohol intake was not allowed to exceed 2 units per day. During testing at baseline and after 12 weeks of exercise, the participants consumed a standardised meal after an overnight fast. A carbohydrate-rich meal including bread, apple juice, cheese and jam was adjusted depending on individual energy requirements and provided $23 \%$ of the estimated total daily energy expenditure 90-120 min prior to the test. Tests were typically performed in the morning; the standardised meal was the only intake after the overnight fast. Water could be consumed freely.

Table 1 Participant characteristics

\begin{tabular}{|c|c|c|c|c|}
\hline \multirow[t]{2}{*}{ Variable } & \multicolumn{2}{|l|}{ Baseline } & \multicolumn{2}{|c|}{ Percentage change after intervention } \\
\hline & Control participants $(n=13)$ & Dysglycaemic men $(n=13)$ & Control participants $(n=13)$ & Dysglycaemic men $(n=13)$ \\
\hline Age (years) & $50 \pm 7$ & $53 \pm 6$ & & \\
\hline \multicolumn{5}{|l|}{ Body composition } \\
\hline Weight (kg) & $78.5 \pm 8.2$ & $95.4 \pm 10.2 *$ & $-0.3 \pm 2.1$ & $-1.7 \pm 2.4^{\dagger}$ \\
\hline BMI $\left(\mathrm{kg} / \mathrm{m}^{2}\right)$ & $23.5 \pm 2.0$ & $29.0 \pm 2.4^{*}$ & $0.0 \pm 2.0$ & $-1.2 \pm 4.5$ \\
\hline FFM $(\mathrm{kg})^{\mathrm{a}}$ & $34.9 \pm 3.5$ & $37.7 \pm 5.0$ & $6.4 \pm 3.8^{\dagger}$ & $5.3 \pm 2.7^{\dagger}$ \\
\hline $\operatorname{scWAT}(\mathrm{kg})^{\mathrm{a}}$ & $10.3 \pm 2.7$ & $18.0 \pm 4.2^{*}$ & $-6.6 \pm 9.2^{\dagger}$ & $-7.3 \pm 6.0^{\dagger}$ \\
\hline $\operatorname{IAAT}(\mathrm{kg})^{\mathrm{a}}$ & $4.0 \pm 2.0$ & $8.8 \pm 2.6^{*}$ & $-16.9 \pm 15.1^{\dagger}$ & $-19.4 \pm 10.8^{\dagger}$ \\
\hline Hepatic fat $(A U)^{b}$ & $2.8 \pm 2.2$ & $9.1 \pm 5.9 *$ & $-23.3 \pm 50.7^{\dagger}$ & $-27.4 \pm 15.8^{\dagger}$ \\
\hline Thigh muscle area $(\mathrm{AU})^{\mathrm{a}}$ & $20.3 \pm 2.9$ & $24.4 \pm 3.1^{*}$ & $9.7 \pm 4.7^{\dagger}$ & $7.1 \pm 6.7^{\dagger}$ \\
\hline \multicolumn{5}{|l|}{ Physical fitness } \\
\hline$\dot{V} \mathrm{O}_{2 \max }\left(\mathrm{ml} \mathrm{kg}^{-1} \min ^{-1}\right)$ & $44.1 \pm 4.4$ & $37.1 \pm 4.9^{*}$ & $13.2 \pm 9.7^{\dagger}$ & $13.3 \pm 7.7^{\dagger}$ \\
\hline Chest press (kg) & $65.6 \pm 16.8$ & $68.7 \pm 13.7$ & $18.4 \pm 8.7^{\dagger}$ & $13.6 \pm 8.4^{\dagger}$ \\
\hline Pull down (kg) & $68.8 \pm 9.3$ & $75.6 \pm 15.1$ & $18.3 \pm 10.1^{\dagger}$ & $13.7 \pm 7.3^{\dagger}$ \\
\hline Leg press (kg) & $199.6 \pm 36.9$ & $248.7 \pm 30.3^{*}$ & $9.8 \pm 7.6^{\dagger}$ & $12.5 \pm 8.4^{\dagger}$ \\
\hline \multicolumn{5}{|l|}{ Glucose metabolism } \\
\hline $\mathrm{HbA}_{1 \mathrm{c}}(\mathrm{mmol} / \mathrm{mol})$ & $33 \pm 4$ & $37 \pm 4 *$ & NA & NA \\
\hline $\mathrm{HbA}_{1 \mathrm{c}}(\%)$ & $5.2 \pm 0.2$ & $5.5 \pm 0.4^{*}$ & NA & NA \\
\hline Fasting glucose (mmol/l) & $5.4 \pm 0.5$ & $5.9 \pm 0.3^{*}$ & $3.1 \pm 4.5^{\dagger}$ & $1.8 \pm 6.8$ \\
\hline Fasting C-peptide (pmol/l) & $588.0 \pm 117.8$ & $932.8 \pm 248.9^{*}$ & $7.3 \pm 23.8$ & $12.3 \pm 45.3$ \\
\hline Fasting insulin (pmol/1) & $38.5 \pm 18.6$ & $65.3 \pm 27.1^{*}$ & $15.1 \pm 49.2$ & $27.6 \pm 66.2$ \\
\hline Plasma NEFA (mmol/l) & $0.3 \pm 0.1$ & $0.2 \pm 0.1$ & $-21.7 \pm 31.1^{\dagger}$ & $16.0 \pm 53.1$ \\
\hline $\operatorname{GIR}\left(\mathrm{mg} \mathrm{kg}^{-1} \mathrm{~min}^{-1}\right)$ & $7.6 \pm 1.6$ & $4.2 \pm 1.8^{*}$ & $37.8 \pm 30.1^{\dagger}$ & $44.4 \pm 58.8^{\dagger}$ \\
\hline \multicolumn{5}{|l|}{ Plasma protein } \\
\hline hsCRP (nmol/l) & $9.5 \pm 7.6$ & $26.7 \pm 21.9$ & $41.0 \pm 96.7$ & $30.1 \pm 121.2$ \\
\hline
\end{tabular}

Data are means $\pm \mathrm{SD}$

Between-group comparisons were performed using unpaired $t$ tests and within-group comparisons were performed using paired $t$ tests. Logarithmic transformation was performed to approximate normal distribution, if necessary, and back-transformed for presentation

${ }^{\text {a }} n=12$ control participants

${ }^{\mathrm{b}} n=10$ control participants and $n=9$ dysglycaemic men

${ }^{*} p<0.05$ between groups (dysglycaemic vs control), ${ }^{\dagger} p<0.05$ baseline vs 12 weeks within group

AU, arbitrary units; FFM, fat free mass; IAAT, intra-abdominal adipose tissue; NA, not available; hsCRP, high-sensitivity C-reactive protein 
a
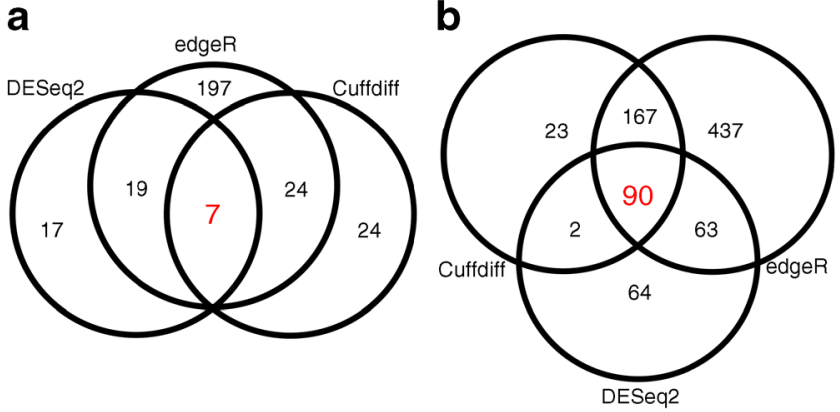

C
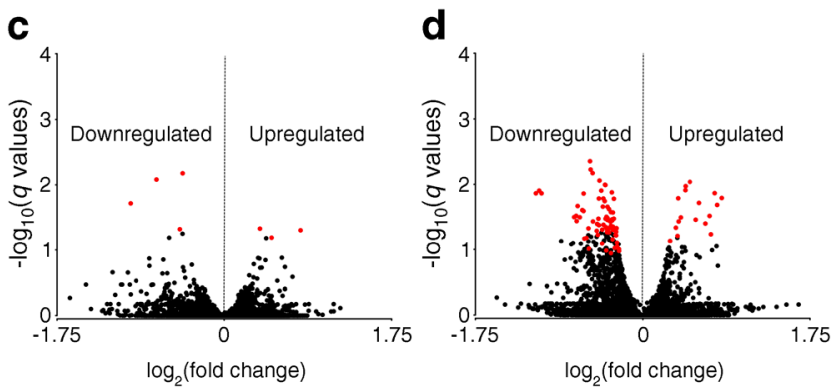

Fig. 2 Exercise-responsive transcripts in scWAT. We compared transcript levels in biopsies obtained from scWAT before and after the 12 week exercise intervention using three commonly applied workflows for mRNA sequencing data: DESeq2, edgeR and Cuffdiff. The results from these three approaches were intersected for both (a) normoglycaemic and (b) dysglycaemic men. Volcano plots show fold changes and $q$ values for (c) normoglycaemic and (d) dysglycaemic men. On the $x$-axis, data points below and above zero indicate downregulation and upregulation, respectively, after the 12 week exercise intervention. On the $y$-axis, large values represent low $q$ values, represented as the mean from all three workflows. The logarithmic transformations used in volcano plots are necessary for comprehensive and symmetrical representation of the large amount of data. Red coloured points indicate statistical significance, whereas black points signifies statistical insignificance within each group, respectively. $q$ values: Benjamini-Hochberg corrected $p$ values

\section{Exercise intervention}

Strength and endurance exercise The participants performed $4 \mathrm{~h}$ of intensive exercise each week for 12 weeks under professional supervision. Two whole-body strength training sessions and two spinning bike interval sessions lasted $1 \mathrm{~h}$ each (Fig. 1). The 12 week intervention included linear progression in workload for both strength and endurance exercises. For strength exercises, during weeks $1-3$ a load that could be lifted a maximum of 12 times (i.e. 12 repetition maximum) was used, which progressed to ten repetition maximum in weeks 4-8 and to eight repetition maximum in weeks 9-12. Abdominal crunches and back extensions were performed with 12-20 repetitions for the whole intervention period. For endurance exercise, during week 1 , three intervals were performed during endurance session one (intervals at $>90 \%$ of maximum heart rate) and six intervals during endurance session two (intervals at $85 \%$ of maximum heart rate) (Fig. 1). For weeks $2-5$, four intervals were performed during session one and seven intervals during session two. For weeks 6-12, five intervals were performed during session one and ten intervals during session two.

\section{Physical fitness and insulin sensitivity}

$\dot{V} \mathrm{O}_{2 \max }$ tests were performed after a standardised warm-up at a workload similar to the final load of an incremental test in which the relationship between work (watt) and oxygen uptake was established. Participants cycled for 1 min followed by a $15 \mathrm{~W}$ increased workload every $30 \mathrm{~s}$ until exhaustion. Test success was based on oxygen consumption increased

Table 2 scWAT transcripts responding to 12 weeks of exercise intervention in control participants

\begin{tabular}{|c|c|c|c|c|c|c|c|c|c|c|c|c|c|}
\hline \multirow[t]{2}{*}{ Gene symbol } & \multirow[t]{2}{*}{ UniProt name } & \multirow[t]{2}{*}{ Secreted? } & \multirow[t]{2}{*}{ RPKM } & \multicolumn{3}{|c|}{ Cuffdiff } & \multicolumn{3}{|l|}{ edgeR } & \multicolumn{4}{|c|}{ DESeq2 } \\
\hline & & & & $\lg _{2} \mathrm{FC}$ & $p$ & FDR & $\lg _{2} \mathrm{FC}$ & $p$ & FDR & $\lg _{2} \mathrm{FC}$ & SE & $p$ & FDR \\
\hline EGFL6 & EGF-like protein 6 & Yes & 19.63 & -0.95 & $<0.01$ & 0.02 & -0.79 & $<0.01$ & $<0.01$ & -0.71 & 0.15 & $<0.01$ & 0.01 \\
\hline UCHL1 & $\begin{array}{l}\text { Ubiquitin carboxyl-terminal } \\
\text { hydrolase }\end{array}$ & WL & 71.69 & -0.48 & $<0.01$ & 0.02 & -0.43 & $<0.01$ & $<0.01$ & -0.43 & 0.08 & $<0.01$ & $<0.01$ \\
\hline CETP & $\begin{array}{l}\text { Cholesteryl ester transfer } \\
\text { protein }\end{array}$ & WL & 12.71 & 0.74 & $<0.01$ & 0.02 & 0.87 & $<0.01$ & $<0.01$ & 0.80 & 0.23 & $<0.01$ & 0.13 \\
\hline$B D K R B 2$ & B2 bradykinin receptor & WL & 3.03 & 0.54 & $<0.01$ & 0.06 & 0.51 & $<0.01$ & $<0.01$ & 0.50 & 0.14 & $<0.01$ & 0.13 \\
\hline OSGIN1 & $\begin{array}{l}\text { Oxidative stress-induced } \\
\text { growth inhibitor } 1\end{array}$ & WL & 12.62 & -0.47 & $<0.01$ & 0.07 & -0.46 & $<0.01$ & $<0.01$ & -0.46 & 0.12 & $<0.01$ & 0.07 \\
\hline$D R D 1$ & $\mathrm{D}(1 \mathrm{~A})$ dopamine receptor & No & 0.85 & -1.15 & $<0.01$ & 0.04 & -1.02 & $<0.01$ & $<0.01$ & -0.98 & 0.23 & $<0.01$ & 0.02 \\
\hline$M E G 3$ & - & No & 30.22 & 0.40 & $<0.01$ & 0.06 & 0.39 & $<0.01$ & $<0.01$ & 0.37 & 0.10 & $<0.01$ & 0.08 \\
\hline
\end{tabular}

Data were modelled as a (paired) before-after design for control participants using three common statistical approaches to mRNA sequencing differential expression analyses (Cuffdiff, edgeR and DESeq2). The FDR was set to $<15 \%$ for each approach, and the results were intersected between all three approaches. MetazSecKB defined secreted proteins

SEs are of the fold change

FDR, false discovery rate; $\lg _{2} \mathrm{FC}, \log _{2}$ of the fold change; RPKM, reads per kilobase per million mapped reads; WL, weakly likely 
Table 3 scWAT transcripts responding to 12 weeks of exercise intervention in men with dysglycaemia

\begin{tabular}{|c|c|c|c|c|c|c|c|c|c|c|c|c|c|}
\hline \multirow[t]{2}{*}{ Gene symbol } & \multirow[t]{2}{*}{ UniProt Name } & \multirow[t]{2}{*}{ Secreted? } & \multirow[t]{2}{*}{ RPKM } & \multicolumn{3}{|c|}{ Cuffdiff } & \multicolumn{3}{|l|}{ edgeR } & \multicolumn{4}{|c|}{ DESeq2 } \\
\hline & & & & $\lg _{2} \mathrm{FC}$ & $p$ & FDR & $\lg _{2} \mathrm{FC}$ & $p$ & FDR & $\lg _{2} \mathrm{FC}$ & $\mathrm{SE}$ & $p$ & FDR \\
\hline SFRP4 & Secreted frizzled-related protein 4 & Yes & 16.57 & -0.54 & $<0.01$ & 0.01 & -0.56 & $<0.01$ & $<0.01$ & -0.55 & 0.12 & $<0.01$ & 0.01 \\
\hline EMILIN2 & $\begin{array}{l}\text { Elastin microfibril interface-located protein } \\
2\end{array}$ & Yes & 16.09 & -0.53 & $<0.01$ & 0.01 & -0.61 & $<0.01$ & $<0.01$ & -0.53 & 0.12 & $<0.01$ & 0.01 \\
\hline ADAMTS4 & $\begin{array}{l}\text { A disintegrin and metalloproteinase with } \\
\text { thrombospondin motifs } 4\end{array}$ & Yes & 5.74 & 0.58 & $<0.01$ & 0.01 & 0.61 & $<0.01$ & $<0.01$ & 0.59 & 0.16 & $<0.01$ & 0.05 \\
\hline$C C L 22$ & C-C motif chemokine 22 & Yes & 2.48 & -0.78 & $<0.01$ & 0.01 & -0.57 & $<0.01$ & 0.05 & -0.57 & 0.17 & $<0.01$ & 0.09 \\
\hline$C 1 Q C$ & $\begin{array}{l}\text { Complement } \mathrm{C} 1 \mathrm{q} \text { subcomponent subunit } \\
\text { C }\end{array}$ & Yes & 132.33 & -0.38 & $<0.01$ & 0.01 & -0.33 & $<0.01$ & 0.01 & -0.33 & 0.10 & $<0.01$ & 0.12 \\
\hline$A N G P T 2$ & Angiopoietin-2 & Yes & 4.86 & -0.64 & $<0.01$ & 0.01 & -0.56 & $<0.01$ & $<0.01$ & -0.55 & 0.11 & $<0.01$ & 0.01 \\
\hline PENK & Proenkephalin-A & Yes & 4.60 & -0.71 & $<0.01$ & 0.01 & -0.65 & $<0.01$ & 0.03 & -0.63 & 0.16 & $<0.01$ & 0.03 \\
\hline$C C L 3$ & C-C motif chemokine 3 & Yes & 4.94 & -0.86 & $<0.01$ & 0.02 & -0.74 & $<0.01$ & 0.01 & -0.72 & 0.21 & $<0.01$ & 0.07 \\
\hline THBS4 & Thrombospondin-4 & Yes & 43.45 & 0.33 & $<0.01$ & 0.02 & 0.38 & $<0.01$ & $<0.01$ & 0.37 & 0.09 & $<0.01$ & 0.02 \\
\hline$T G F B I$ & TGF- $\beta$-induced protein ig-h3 & Yes & 49.74 & -0.32 & $<0.01$ & 0.03 & -0.30 & $<0.01$ & 0.02 & -0.30 & 0.08 & $<0.01$ & 0.05 \\
\hline NPTX2 & Neuronal pentraxin-2 & Yes & 0.72 & 0.76 & $<0.01$ & 0.06 & 0.72 & $<0.01$ & 0.01 & 0.70 & 0.18 & $<0.01$ & 0.02 \\
\hline STC2 & Stanniocalcin-2 & Yes & 6.10 & -0.33 & $<0.01$ & 0.07 & -0.33 & $<0.01$ & $<0.01$ & -0.32 & 0.09 & $<0.01$ & 0.07 \\
\hline $\mathrm{CFH}$ & Complement factor $\mathrm{H}$ & Yes & 55.64 & -0.31 & $<0.01$ & 0.10 & -0.28 & $<0.01$ & 0.04 & -0.29 & 0.08 & $<0.01$ & 0.04 \\
\hline TNFRSF11B & TNF receptor superfamily member $11 \mathrm{~B}$ & Yes & 4.25 & -0.44 & $<0.01$ & 0.12 & -0.43 & $<0.01$ & 0.05 & -0.43 & 0.12 & $<0.01$ & 0.07 \\
\hline$F B L N 1$ & Fibulin-1 & HL & 73.17 & -0.33 & $<0.01$ & 0.01 & -0.32 & $<0.01$ & 0.01 & -0.32 & 0.08 & $<0.01$ & 0.02 \\
\hline$F S T$ & FST protein & HL & 10.73 & 0.43 & $<0.01$ & 0.01 & 0.45 & $<0.01$ & $<0.01$ & 0.45 & 0.11 & $<0.01$ & 0.02 \\
\hline$B M P 3$ & Bone morphogenetic protein $3 \mathrm{~F}$ & $\mathrm{HL}$ & 0.60 & 0.82 & $<0.01$ & 0.01 & 0.79 & $<0.01$ & $<0.01$ & 0.75 & 0.20 & $<0.01$ & 0.03 \\
\hline SLAMF7 & SLAM family member $7 \mathrm{~F}$ & $\mathrm{HL}$ & 3.47 & -0.72 & $<0.01$ & 0.01 & -0.63 & $<0.01$ & $<0.01$ & -0.62 & 0.16 & $<0.01$ & 0.03 \\
\hline$H B A 2$ & $\alpha-2$ globin $\mathrm{F}$ & $\mathrm{HL}$ & 50.28 & -1.35 & $<0.01$ & 0.01 & -1.22 & $<0.01$ & $<0.01$ & -1.12 & 0.29 & $<0.01$ & 0.03 \\
\hline$C D 4$ & $\mathrm{CD} 4$ protein & $\mathrm{HL}$ & 26.32 & -0.39 & $<0.01$ & 0.01 & -0.33 & $<0.01$ & $<0.01$ & -0.33 & 0.10 & $<0.01$ & 0.07 \\
\hline LIPA & Lysosomal acid lipase $\mathrm{F}$ & $\mathrm{HL}$ & 139.10 & -0.65 & $<0.01$ & 0.01 & -0.52 & $<0.01$ & $<0.01$ & -0.52 & 0.16 & $<0.01$ & 0.10 \\
\hline$P D E 11 A$ & $\begin{array}{l}\text { Dual 3',5'-cyclic-AMP and -GMP } \\
\text { phosphodiesterase 11A }\end{array}$ & $\mathrm{HL}$ & 2.43 & 0.42 & $<0.01$ & 0.02 & 0.49 & $<0.01$ & $<0.01$ & 0.49 & 0.11 & $<0.01$ & 0.01 \\
\hline ITGAL & Integrin $\alpha-\mathrm{L}$ & $\mathrm{HL}$ & & -0.45 & $<0.01$ & 0.02 & -0.37 & $<0.01$ & $<0.01$ & -0.37 & 0.11 & $<0.01$ & 0.08 \\
\hline ADAMTS18 & $\begin{array}{l}\text { rin and metalloproteinase with } \\
\text { sspondin motifs } 18\end{array}$ & $\mathrm{HL}$ & 1.03 & 0.55 & $<0.01$ & 0.02 & 0.58 & $<0.01$ & $<0.01$ & 0.56 & 0.16 & $<0.01$ & 0.08 \\
\hline LILRA2 & -like receptor subfamily A & $\mathrm{HL}$ & 2.12 & -0.73 & $<0.01$ & 0.03 & -0.67 & $<0.01$ & 0.03 & 66 & 0.18 & $<0.01$ & 0.04 \\
\hline$C D 86$ & T-lymphocyte activation antigen CD86 F & $\mathrm{HL}$ & & -0.51 & & 0.03 & -0.42 & $<0.01$ & 0 & -0.43 & 12 & $<0.01$ & 0.05 \\
\hline GALNT6 & lgalactosaminyltransferase $6 \mathrm{~F}$ & $\mathrm{HL}$ & 1.01 & -0 & $<0.01$ & 0.04 & -0 & $<0.01$ & 0.02 & 69 & 0.19 & $<0.01$ & 0.05 \\
\hline PILRA & Paired Ig-like type 2 receptor $\alpha$ & $\mathrm{HL}$ & & -0.49 & $<0.01$ & 0.04 & -0.49 & $<0.01$ & 0.01 & -0.48 & .14 & $<0.01$ & 0.08 \\
\hline$G R N$ & Granulins F & $\mathrm{HL}$ & 182.11 & -0.32 & $<0.01$ & 0.06 & -0.28 & $<0.01$ & 0.01 & -0.28 & 0.08 & $<0.01$ & 0.09 \\
\hline$A C E$ & $\mathrm{ACE}$ & $\mathrm{HL}$ & 18.74 & -0.29 & $<0.01$ & 0.07 & -0.27 & $<0.01$ & 0.02 & -0.28 & 0.08 & $<0.01$ & 0.05 \\
\hline PTPRJ & $\begin{array}{r}\text { Receptc } \\
\text { phos }\end{array}$ & $\mathrm{HL}$ & 5.02 & -0.31 & $<0.01$ & 0.08 & -0.31 & $<0.01$ & $<0.01$ & -0.31 & 0.09 & $<0.01$ & 0.07 \\
\hline$T S K U$ & Tsukus & $\mathrm{HL}$ & - & 0.31 & 1 & 0.09 & 0.2 & $<0.01$ & $<0.01$ & 0.28 & 0.09 & $<0.01$ & 0.13 \\
\hline PAMRI & Inactiv & $\mathrm{HL}$ & 5.82 & -0.39 & $<0.01$ & 0.09 & -0.41 & $<0.01$ & $<0.01$ & -0.41 & 0.11 & $<0.01$ & 0.03 \\
\hline$C D 22$ & $\mathrm{~B}$ cell re & $\mathrm{HL}$ & 1.19 & -0.62 & $<0.01$ & 0.12 & -0.56 & $<0.01$ & 0.12 & -0.57 & 0.16 & $<0.01$ & 0.05 \\
\hline$B C A T 1$ & Alternative protein BCAT1 & $\mathrm{L}$ & 2.90 & -0.50 & $<0.01$ & 0.01 & -0.43 & $<0.01$ & $<0.01$ & -0.42 & 0.12 & $<0.01$ & 0.06 \\
\hline FCERIG & High-affinity Ig epsilon receptor subunit $\gamma$ & $\mathrm{L}$ & 92.32 & -0.56 & $<0.01$ & 0.01 & -0.50 & $<0.01$ & $<0.01$ & -0.48 & 0.16 & $<0.01$ & 0.15 \\
\hline TBXAS1 & Thromboxane-A synthase & $\mathrm{L}$ & 6.95 & -0.46 & $<0.01$ & 0.02 & -0.40 & $<0.01$ & $<0.01$ & -0.40 & 0.10 & $<0.01$ & 0.03 \\
\hline ITGAM & Integrin $\alpha-\mathrm{M} \mathrm{F}$ & WL & 9.36 & -0.53 & $<0.01$ & 0.01 & -0.46 & $<0.01$ & $<0.01$ & -0.45 & 0.11 & $<0.01$ & 0.02 \\
\hline$L B H$ & Protein LBH & WL & 11.35 & -0.42 & $<0.01$ & 0.01 & -0.40 & $<0.01$ & $<0.01$ & -0.40 & 0.10 & $<0.01$ & 0.02 \\
\hline$G M 2 A$ & Ganglioside GM2 & WL & 35.68 & -0.45 & $<0.01$ & 0.01 & -0.39 & $<0.01$ & $<0.01$ & -0.39 & 0.10 & $<0.01$ & 0.02 \\
\hline$H B B$ & Mutant $\beta$-globin & WL & 343.81 & -1.29 & $<0.01$ & 0.01 & -1.12 & $<0.01$ & $<0.01$ & -1.06 & 0.28 & $<0.01$ & 0.03 \\
\hline$K Y N U$ & Kynureninase & WL & 2.70 & -0.81 & $<0.01$ & 0.01 & -0.69 & $<0.01$ & 0.02 & -0.68 & 0.18 & $<0.01$ & 0.03 \\
\hline PTPN6 & $\begin{array}{l}\text { Tyrosine-protein phosphatase non-receptor } \\
\text { type } 6\end{array}$ & WL & 16.46 & -0.48 & $<0.01$ & 0.01 & -0.42 & $<0.01$ & $<0.01$ & -0.41 & 0.11 & $<0.01$ & 0.04 \\
\hline NDRG4 & Protein NDRG4 F & WL & & 0.7 & $<0.01$ & 0.01 & 0.8 & $<0.01$ & $<0.01$ & 0 & 0.22 & $<0.01$ & 0.05 \\
\hline ARHGAP30 & Rho GTPase-activating & WL & 9.27 & -0.45 & $<0.01$ & 0.01 & -0.39 & $<0.01$ & $<0.01$ & -0.38 & 0.11 & $<0.01$ & 0.09 \\
\hline LGALS9 & Galectin F & WL & 30.71 & -0.43 & $<0.01$ & 0.01 & -0.39 & $<0.01$ & $<0.01$ & -0.39 & 0.12 & $<0.01$ & 0.09 \\
\hline$L S P 1$ & Lymphocyt & WL & 99.05 & -0.34 & $<0.01$ & 0.01 & -0.31 & $<0.01$ & 0.01 & -0.31 & 0.10 & $<0.01$ & 0.11 \\
\hline$S T X B P 2$ & Syntaxin-binding protein $2 \mathrm{~F}$ & WL & 8.19 & -0.50 & $<0.01$ & 0.01 & -0.39 & $<0.01$ & $<0.01$ & -0.39 & 0.12 & $<0.01$ & 0.12 \\
\hline$T R P V 2$ & $\begin{array}{l}\text { Transient receptor potential cation channel } \\
\text { subfamily V member } 2 \mathrm{~F}\end{array}$ & WL & 14.33 & -0.38 & $<0.01$ & 0.02 & -0.34 & $<0.01$ & $<0.01$ & -0.33 & 0.10 & $<0.01$ & 0.09 \\
\hline DOK2 & Dock & $\mathrm{W}$ & & -0 & & 0.02 & -0.36 & & & -0.35 & 0.11 & $<0.01$ & 0.14 \\
\hline SOX9 & SRY-t & WL & 3.39 & 0.4 & $<0.01$ & 0.03 & 0.45 & $<0.01$ & $<0.01$ & 0.44 & 0.09 & $<0.01$ & 0.01 \\
\hline DHCR24 & $\operatorname{Delta}(24)$-ster & WL & 44.49 & & & 0.03 & -0.32 & $<0.01$ & 0.01 & -0.32 & 0.09 & $<0.01$ & 0.06 \\
\hline$P A Q R 5$ & Alternative protein PAQR5 & WL & 0.72 & -0.70 & $<0.01$ & 0.04 & -0.63 & $<0.01$ & 0.06 & -0.61 & 0.19 & $<0.01$ & 0.10 \\
\hline
\end{tabular}


Table 3 (continued)

\begin{tabular}{|c|c|c|c|c|c|c|c|c|c|c|c|c|c|}
\hline \multirow[t]{2}{*}{ Gene symbol } & \multirow[t]{2}{*}{ UniProt Name } & \multirow[t]{2}{*}{ Secreted? } & \multirow[t]{2}{*}{ RPKM } & \multicolumn{3}{|c|}{ Cuffdiff } & \multicolumn{3}{|l|}{ edgeR } & \multicolumn{4}{|c|}{ DESeq2 } \\
\hline & & & & $\lg _{2} \mathrm{FC}$ & $p$ & FDR & $\lg _{2} \mathrm{FC}$ & $p$ & FDR & $\lg _{2} \mathrm{FC}$ & $\mathrm{SE}$ & $p$ & FDR \\
\hline CHST15 & Alternative protein CHST15 & WL & 5.47 & -0.38 & $<0.01$ & 0.04 & -0.37 & $<0.01$ & $<0.01$ & -0.37 & 0.09 & $<0.01$ & 0.02 \\
\hline C8orf4 & Alternative protein C8orf4 & WL & 6.84 & 0.45 & $<0.01$ & 0.04 & 0.37 & $<0.01$ & $<0.01$ & 0.36 & 0.12 & $<0.01$ & 0.14 \\
\hline$R O R C$ & $\begin{array}{l}\text { RAR-related orphan receptor C, isoform } \\
\text { CRA_a }\end{array}$ & WL & 0.45 & 0.78 & $<0.01$ & 0.06 & 0.76 & $<0.01$ & $<0.01$ & 0.71 & 0.22 & $<0.01$ & 0.11 \\
\hline MYOIE & Unconventional myosin-Ie F & WL & 8.42 & -0.32 & $<0.01$ & 0.07 & -0.31 & $<0.01$ & $<0.01$ & -0.31 & 0.07 & $<0.01$ & 0.01 \\
\hline RABIIFIP4 & Rab11 family-interacting protein $4 \mathrm{~F}$ & WL & 0.43 & -0.66 & $<0.01$ & 0.09 & -0.59 & $<0.01$ & 0.09 & -0.59 & 0.14 & $<0.01$ & 0.02 \\
\hline$P L X N C 1$ & Plexin-C1 & WL & 2.47 & -0.44 & $<0.01$ & 0.14 & -0.38 & $<0.01$ & 0.04 & -0.38 & 0.12 & $<0.01$ & 0.13 \\
\hline$R C A N 2$ & Calcipressin-2 & WL & 9.45 & -0.30 & $<0.01$ & 0.15 & -0.29 & $<0.01$ & $<0.01$ & -0.29 & 0.10 & $<0.01$ & 0.14 \\
\hline$C Y B A$ & Cytochrome b-245 light chain F & WL & 163.49 & -0.27 & $<0.01$ & 0.15 & -0.25 & $<0.01$ & 0.05 & -0.25 & 0.08 & $<0.01$ & 0.10 \\
\hline PSD4 & $\begin{array}{l}\mathrm{PH} \text { and SEC7 domain-containing protein } 4 \\
\text { F }\end{array}$ & WL & 2.38 & -0.39 & $<0.01$ & 0.15 & -0.33 & $<0.01$ & 0.06 & -0.33 & 0.11 & $<0.01$ & 0.13 \\
\hline$A B C B 11$ & Bile salt export pump & No & 0.49 & 0.71 & $<0.01$ & 0.06 & 0.68 & $<0.01$ & 0.03 & 0.66 & 0.17 & $<0.01$ & 0.03 \\
\hline$A D R A 2 A$ & $\alpha-2 \mathrm{~A}$ adrenergic receptor & No & 50.86 & -0.29 & $<0.01$ & 0.05 & -0.28 & $<0.01$ & 0.04 & -0.28 & 0.09 & $<0.01$ & 0.09 \\
\hline$A R H G D I B$ & Rho GDP-dissociation inhibitor 2 & No & 133.16 & -0.28 & $<0.01$ & 0.09 & -0.27 & $<0.01$ & 0.05 & -0.28 & 0.05 & $<0.01$ & $<0.01$ \\
\hline$C D 180$ & CD180 antigen & No & 1.39 & -0.86 & $<0.01$ & 0.02 & -0.71 & $<0.01$ & 0.02 & -0.70 & 0.19 & $<0.01$ & 0.05 \\
\hline CD53 & Leucocyte surface & No & 23.25 & -0.57 & $<0.01$ & 0.01 & -0.48 & $<0.01$ & $<0.01$ & -0.46 & 0.12 & $<0.01$ & 0.04 \\
\hline COROIA & Coron & No & 16.74 & -0.39 & $<0.01$ & 0.02 & -0.36 & $<0.01$ & $<0.01$ & -0.35 & 0.10 & $<0.01$ & 0.05 \\
\hline CTSZ & Cathe & No & 275.21 & -0.28 & $<0.01$ & 0.11 & -0.25 & $<0.01$ & 0.05 & -0.25 & 0.08 & $<0.01$ & 0.15 \\
\hline$C Y B B$ & Cytoc & No & 11.44 & -0.50 & $<0.01$ & 0.01 & -0.44 & $<0.01$ & $<0.01$ & -0.43 & 0.11 & $<0.01$ & 0.03 \\
\hline CYSLTR2 & Cyste & No & 0.56 & 0.84 & $<0.01$ & 0.04 & 0.87 & $<0.01$ & $<0.01$ & 0.83 & 0.18 & $<0.01$ & 0.01 \\
\hline$A C K R 1$ & 1 chemokine 1 & No & 101.21 & -0.31 & $<0.01$ & 0.08 & -0.27 & $<0.01$ & 0.06 & -0.27 & 0.09 & $<0.01$ & 0.13 \\
\hline DOCK2 & of cytokinesis protein 2 & No & 3.87 & -0.37 & $<0.01$ & 0.07 & -0.32 & $<0.01$ & $<0.01$ & -0.32 & 0.10 & $<0.01$ & 0.14 \\
\hline FPR3 & 1 peptide receptor 3 & No & 8.8 & -0.43 & $<0.01$ & 0.02 & -0.39 & $<0.01$ & $<0.01$ & -0.37 & 0.12 & $<0.01$ & 0.14 \\
\hline$G S D M B$ & Gasde & No & 10.92 & 0.45 & $<0.01$ & 0.01 & 0.38 & $<0.01$ & $<0.01$ & 0.37 & 0.11 & $<0.01$ & 0.10 \\
\hline$H B A 1$ & Haemoglobin st & No & 11.41 & -1.27 & $<0.01$ & 0.01 & -1.17 & $<0.01$ & $<0.01$ & -1.08 & 0.28 & $<0.01$ & 0.03 \\
\hline IFITM1 & IFN-induced transmembrane $\mathrm{p}$ & No & 208.95 & -0.36 & $<0.01$ & 0.01 & -0.30 & $<0.01$ & 0.01 & -0.30 & 0.08 & $<0.01$ & 0.02 \\
\hline KCNJ5 & $\begin{array}{l}\text { G protein-activated inward rectifier } \\
\text { potassium channel } 4\end{array}$ & No & 2.18 & -0.80 & $<0.01$ & 0.01 & -0.62 & $<0.01$ & 0.03 & -0.62 & 0.17 & $<0.01$ & 0.04 \\
\hline LHFPL2 & $\begin{array}{l}\text { LHFPL tetraspan subfamily member } 2 \\
\text { protein }\end{array}$ & No & 9.84 & -0.28 & $<0.01$ & 0.15 & -0.27 & $<0.01$ & $<0.01$ & -0.27 & 0.08 & $<0.01$ & 0.09 \\
\hline MAPK13 & $\begin{array}{l}\text { Mitogen-activated protein kinase } \\
\text { 13Mitogen-activated protein kinase }\end{array}$ & No & 2.29 & -0.48 & 0.01 & 0.01 & -0.47 & $<0.01$ & 0.04 & -0.47 & 0.15 & $<0.01$ & 0.13 \\
\hline$M A R C O$ & Macrophage receptor MARCO & No & 13.15 & -0.36 & $<0.01$ & 0.08 & -0.34 & $<0.01$ & $<0.01$ & -0.34 & 0.09 & $<0.01$ & 0.03 \\
\hline NUP210 & Nuclear pore membrane glycoprotein 210 & No & 1.17 & -0.53 & $<0.01$ & 0.04 & -0.46 & $<0.01$ & 0.06 & -0.46 & 0.11 & $<0.01$ & 0.02 \\
\hline PKD1L2 & Polycystic kidney disease protein 1 -like 2 & No & 11.89 & 0.36 & $<0.01$ & 0.06 & 0.35 & $<0.01$ & $<0.01$ & 0.34 & 0.10 & $<0.01$ & 0.08 \\
\hline PLEK & Pleckstrin & No & 15.44 & -0.50 & $<0.01$ & 0.01 & -0.38 & $<0.01$ & $<0.01$ & -0.37 & 0.12 & $<0.01$ & 0.14 \\
\hline$R A S S F 2$ & $\begin{array}{l}\text { Ras association domain-containing protein } \\
\quad 2\end{array}$ & No & 6.06 & -0.33 & $<0.01$ & 0.08 & -0.32 & $<0.01$ & $<0.01$ & -0.32 & 0.08 & $<0.01$ & 0.02 \\
\hline SAMHD1 & $\begin{array}{l}\text { Deoxynucleoside triphosphate } \\
\text { triphosphohydrolase SAMHD1 }\end{array}$ & No & 32.08 & -0.27 & $<0.01$ & 0.12 & -0.28 & $<0.01$ & 0.02 & -0.28 & 0.08 & $<0.01$ & 0.04 \\
\hline SASH3 & $\begin{array}{l}\text { SAM and SH3 domain-containing protein } \\
3\end{array}$ & No & 5.91 & -0.58 & $<0.01$ & 0.01 & -0.48 & $<0.01$ & $<0.01$ & -0.46 & 0.14 & $<0.01$ & 0.09 \\
\hline SCARA3 & Scavenger receptor class A member 3 & No & 13.86 & -0.36 & $<0.01$ & 0.02 & -0.36 & $<0.01$ & $<0.01$ & -0.36 & 0.11 & $<0.01$ & 0.12 \\
\hline SLC25A18 & Mitochondrial glutamate carrier 2 & No & 5.65 & 0.40 & $<0.01$ & 0.09 & 0.40 & $<0.01$ & $<0.01$ & 0.40 & 0.08 & $<0.01$ & 0.01 \\
\hline$S Y K$ & Tyrosine-protein kinase SYK & No & 5.18 & -0.42 & $<0.01$ & 0.01 & -0.38 & $<0.01$ & $<0.01$ & -0.37 & 0.11 & $<0.01$ & 0.09 \\
\hline
\end{tabular}

Data were modelled as a (paired) before-after design for men with dysglycaemia using three common statistical approaches to mRNA sequencing differential expression analyses (Cuffdiff, edgeR and DESeq2). The FDR was set to $<15 \%$ for each approach, and the results were intersected between all three approaches. MetazSecKB defined secreted proteins

SEs are of the fold change

F, fragment; FDR, false discovery rate; HL, highly likely; L, likely; $\lg _{2} \mathrm{FC}, \log _{2}$ of the fold change; RPKM, reads per kilobase per million mapped reads; WL, weakly likely

by $<0.5 \mathrm{ml} \mathrm{kg}^{-1} \mathrm{~min}^{-1}$ over a $30 \mathrm{~W}$ increase in workload, respiratory exchange ratio values $>1.10$ and blood lactate $>7.0$ $\mathrm{mmol} / 1$.

Euglycaemic-hyperinsulinaemic clamp Euglycaemichyperinsulinaemic clamps were performed after an overnight fast. A fixed dose of insulin $40 \mathrm{mU} \mathrm{m}^{-2} \mathrm{~min}^{-1}$ was infused, and glucose $(200 \mathrm{mg} / \mathrm{ml})$ was injected to maintain euglycaemia ( $5.0 \mathrm{mmol} / \mathrm{l})$ for $150 \mathrm{~min}$ [29]. Insulin sensitivity is reported as the glucose infusion rate (GIR) during the last $30 \mathrm{~min}$ relative to body weight. The whole blood glucose concentration was measured using a glucose oxidase method 
(YSI 2300, YSI, Yellow Springs, OH, USA) and the plasma glucose concentration was calculated as whole blood glucose $\times 1.119$.

\section{MRI/MRS}

MRI methods were used to quantify fat mass [26]. The ankle-to-neck MRI protocol included a 3D Dixon acquisition providing water and lipid quantification. Data were then analysed using the nordicICE software package (NordicNeuroLab, Bergen, Norway) and the jMRUI v.5.2 [30] software package.

\section{Tissue sampling}

As described previously [26], we obtained scWAT, skeletal muscle biopsies and blood samples before and after the 12 week exercise intervention (Fig. 1). Biopsies were obtained from the periumbilical subcutaneous tissue and from the vastus lateralis muscle. After sterilisation, a lidocaine-based local anaesthetic was injected into the skin and subcutis prior to both skeletal muscle and scWAT biopsies [26]. Biopsies were dissected on a cold aluminium plate to remove blood and other materials before freezing.

\section{Transcriptomics}

Biopsies were frozen in liquid nitrogen, crushed to powder with a pestle in a liquid nitrogen-cooled mortar, transferred into $1 \mathrm{ml}$ QIAzol Lysis Reagent (Qiagen, Hilden, Germany) and homogenised using TissueRuptor (Qiagen) at full speed for $15 \mathrm{~s}$, twice. Total RNA was isolated from the homogenate using the miRNeasy Mini Kit (Qiagen). RNA integrity and concentration were determined using Agilent RNA 6000 Nano Chips on a Bioanalyzer 2100 (Agilent Technologies, Santa Clara, CA, USA). RNA was converted to cDNA using the High-Capacity cDNA Reverse Transcription Kit (Applied Biosystems, Foster, CA, USA). The cDNA reaction mixture was diluted in water and a cDNA equivalent of 25 ng RNA was used for each sample.

TaqMan real-time quantitative RT-PCR Quantitative real-time PCR was performed with reagents and instruments from Applied Biosystems in the 96-well format using a 7900HT Fast instrument and SDS 2.3 software (Applied Biosystems). Predeveloped primers and probe sets (TaqMan assays, Applied Biosystems) were used to analyse mRNA levels of SFRP4 (Hs00180066_m1), LEP (Hs00174877_m1), TNFRSF11B (Hs00900358_m1), IL6 (Hs00985639_m1) and ADIPOQ

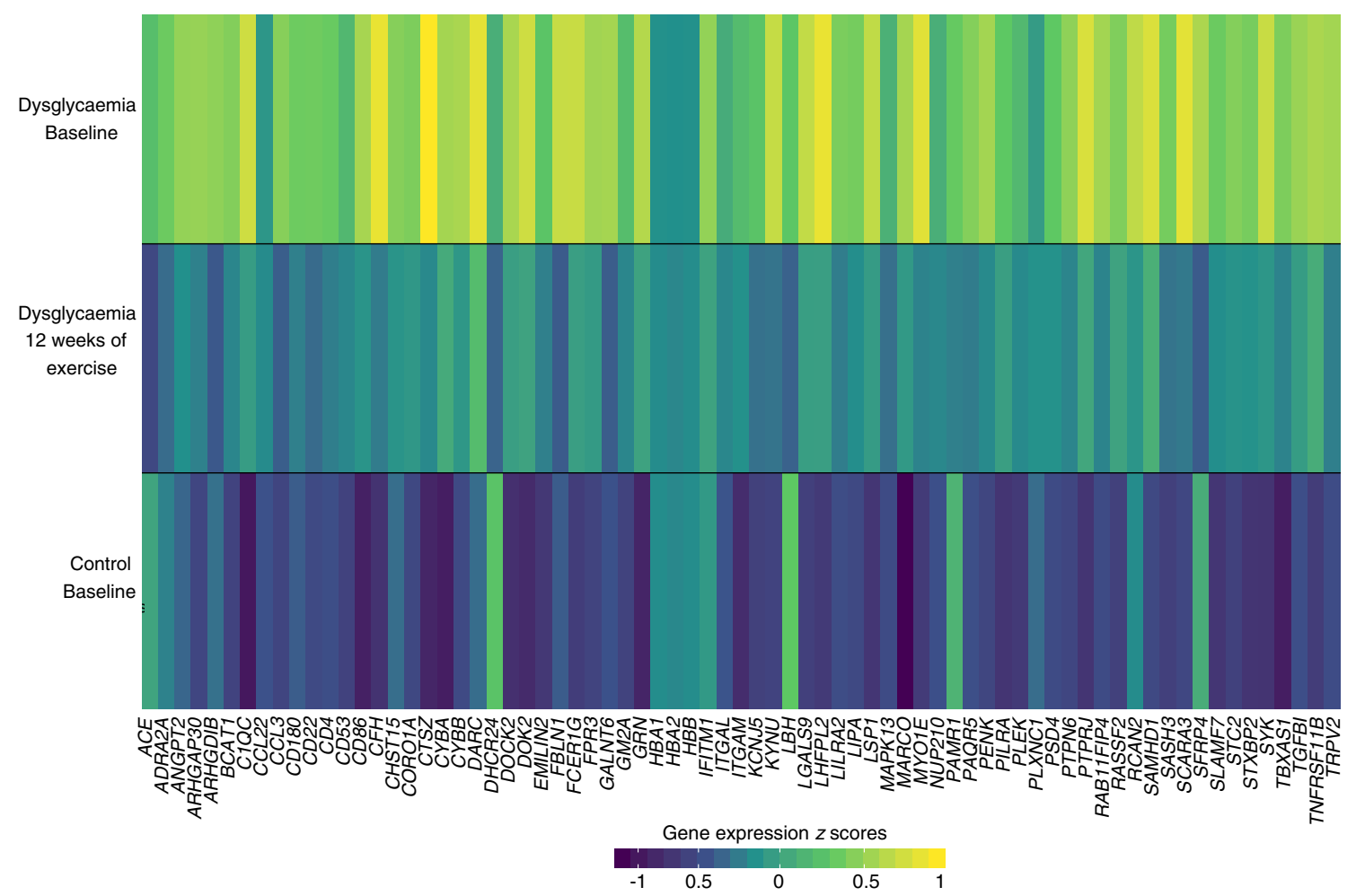

Fig. 3 Twelve weeks of exercise normalised enhanced transcript levels in median level across all participants in the specified group, and each column represents one gene. The scale bar below the heat map represents centred and scaled reads per kilobase of transcript per million mapped reads 
Table 4 mRNA sequencing compared with RT-PCR

\begin{tabular}{|c|c|c|c|c|}
\hline \multirow[t]{2}{*}{ Gene } & \multicolumn{2}{|c|}{ Control men } & \multicolumn{2}{|c|}{ Men with dysglycaemia } \\
\hline & $\lg _{2} \mathrm{FC}$ & $p$ value & $\lg _{2} \mathrm{FC}$ & $p$ value \\
\hline \multicolumn{5}{|l|}{$A D I P O Q$} \\
\hline RT-PCR & -0.152 & 0.635 & -0.022 & 0.910 \\
\hline Cuffdiff & 0.000 & 1.000 & 0.000 & 1.000 \\
\hline DESeq2 & 0.066 & 0.379 & 0.086 & 0.239 \\
\hline edgeR & 0.067 & 0.464 & 0.086 & 0.291 \\
\hline \multicolumn{5}{|l|}{$L E P$} \\
\hline RT-PCR & -0.222 & 0.015 & -0.278 & 0.122 \\
\hline Cuffdiff & -0.153 & 0.333 & -0.294 & 0.015 \\
\hline DESeq2 & -0.146 & 0.057 & -0.296 & 0.002 \\
\hline edgeR & -0.145 & 0.086 & -0.299 & 0.002 \\
\hline \multicolumn{5}{|l|}{ IL6 } \\
\hline RT-PCR & -0.144 & 0.510 & 0.018 & 0.965 \\
\hline Cuffdiff & 0.251 & 0.496 & 0.799 & 0.006 \\
\hline DESeq2 & 0.057 & 0.838 & 0.401 & 0.121 \\
\hline edgeR & 0.041 & 0.886 & 0.395 & 0.139 \\
\hline \multicolumn{5}{|l|}{ SFRP4 } \\
\hline RT-PCR & -0.374 & 0.052 & -0.495 & 0.004 \\
\hline Cuffdiff & -0.341 & 0.005 & -0.536 & $<0.001$ \\
\hline DESeq2 & -0.388 & 0.052 & -0.553 & $<0.001$ \\
\hline edgeR & -0.418 & 0.013 & -0.563 & $<0.001$ \\
\hline
\end{tabular}

Three statistical approaches to mRNA sequencing differential expression analyses were compared with each other, as well with results obtained using quantitative RT-PCR.

$\lg _{2} \mathrm{FC}, \log _{2}$ of the fold change

(Hs00605917_m1). Relative target mRNA levels were calculated as $2^{-\Delta \mathrm{C}_{\mathrm{t}}}$ and normalised to $B 2 M\left(\mathrm{Hs} 00984230 \_\mathrm{ml}\right)$.

Microarrays Purified RNA was labelled using the Affymetrix WT PLUS reagent kit (Affymetrix, Santa Clara, CA, USA) and hybridised to an Affymetrix Human Gene 1.1 ST array plate. Hybridisation, washing and scanning were carried out on the Affymetrix GeneTitan platform according to the manufacturer's instructions. Arrays were analysed using the $\mathrm{R}$ package Oligo v.1.46 [31] following standard procedures for quality checks and calculation of normalised expression values.

High-throughput mRNA sequencing All muscle and scWAT samples were deep-sequenced using the Illumina HiSeq 2000 system (San Diego, CA, USA) with multiplex at the Norwegian Sequencing Centre, University of Oslo. Illumina HiSeq RTA (real-time analysis) v1.17.21.3 was used. Reads passing Illumina's recommended parameters were demultiplexed using CASAVA v1.8.2 (Illumina). For pre-alignment quality checks, we used the software FastQC v0.10.1 (Illumina). The mean library size was approximately 44 million unstranded $51 \mathrm{bp}$ single-ended reads for muscle tissue and approximately 52 million for scWAT, with no differences between groups or time points. No batch effects were present. cDNA sequenced reads alignment was performed using Tophat v2.0.8 [32], Samtools v0.1.18 [33] and Bowtie v2.1.0 [34] with default settings against the UCSC hg19 (University of California Santa Cruz, Santa Cruz, CA, USA) annotated transcriptome and genome dated 14 May 2013. Postalignment quality controls were performed using the Integrative Genome Viewer v2.3 (Illumina) and BED tools v2.19.1 [35]. Reads were counted using the intersection strict mode in HTSeq v0.6.1 [36].

Differential transcript expression using mRNA sequencing The edgeR v3.4.2 [37], DESeq2 v1.4.5 [38], and Cuffdiff v2.1.1 [32] workflows were performed. Statistical significance was set at a false discovery rate of $<15 \%$ for each approach, and then intersected to find coherent results from the three approaches. TaqMan real-time RT-PCR and microarrays were subsequently used to validate the results. Expression levels are presented as reads per kilobase of transcript per million mapped reads.

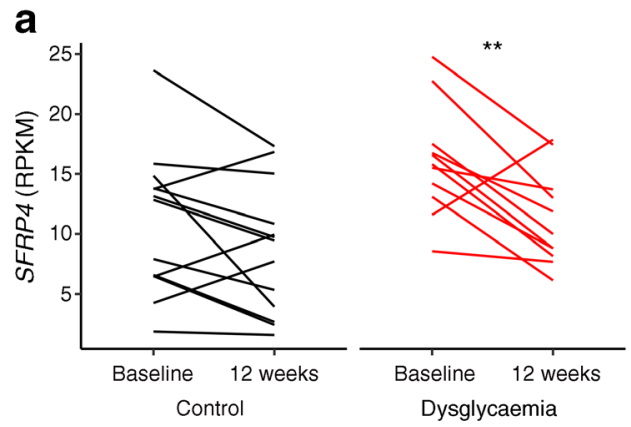

Fig. 4 Validation of mRNA sequencing results. We analysed the transcript most downregulated in men with dysglycaemia after 12 weeks of exercise (SFRP4; see Table 3) using (a) mRNA sequencing $(-28.9 \%[p>0.05]$ and $-67.8 \%\left[{ }^{* *} p<0.01\right]$ in control individuals and men with dysglycaemia, b

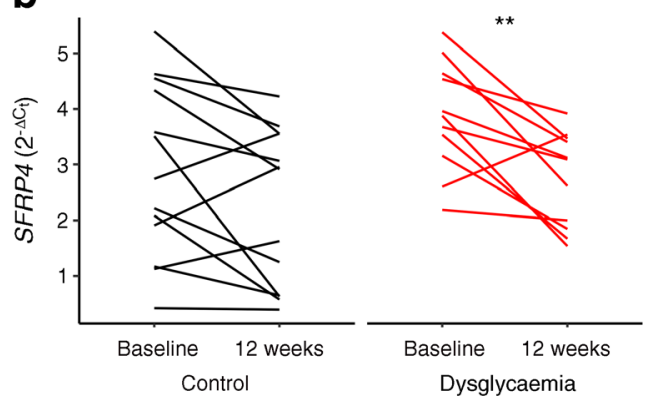

respectively) and (b) real-time quantitative RT-PCR $(-66.1 \%[p>0.05]$ and $-112.4 \%[* * p<0.01]$ in control individuals and men with dysglycaemia, respectively). Paired $t$ tests were used for comparisons. RPKM, reads per kilobase of transcript per million mapped reads 

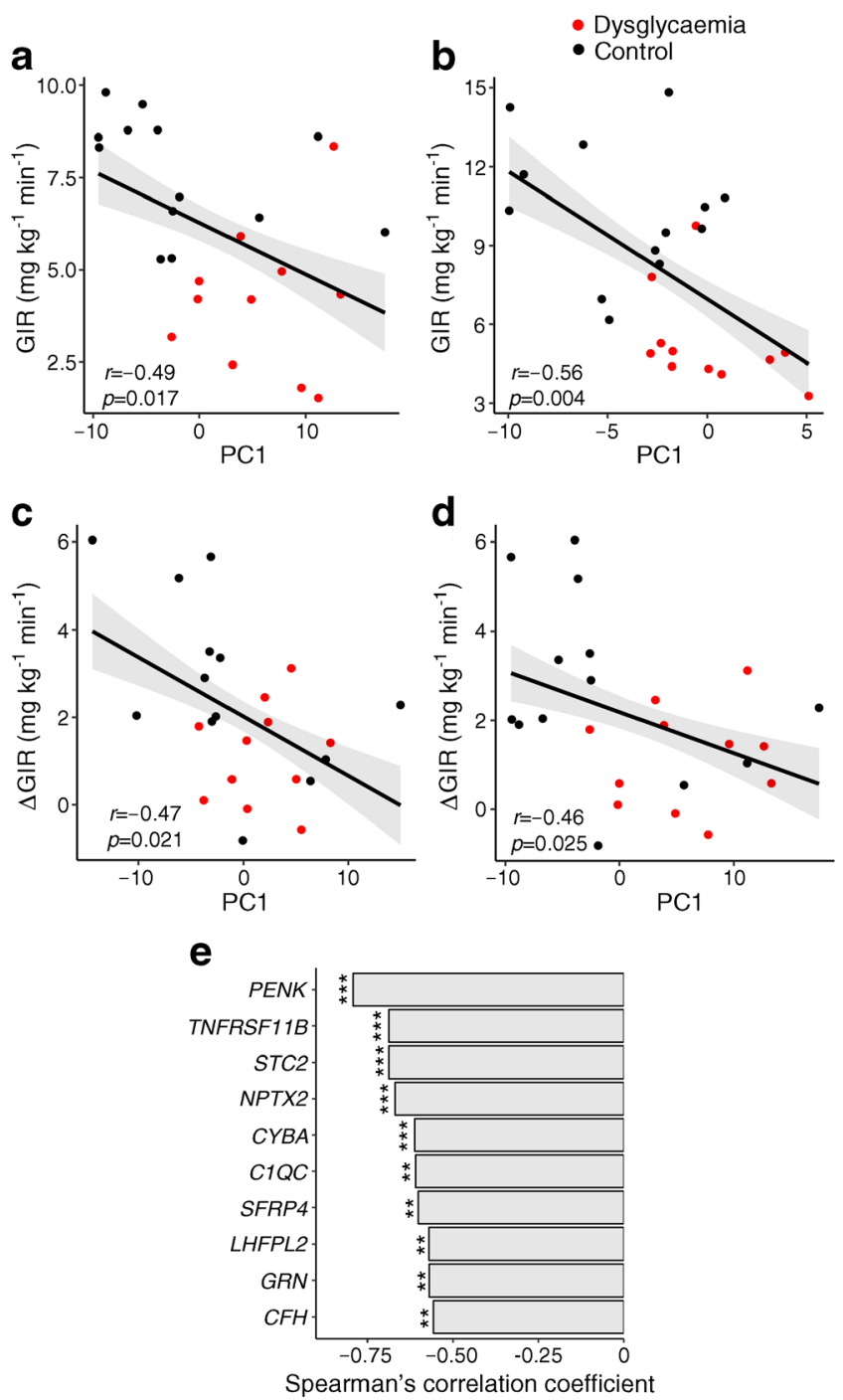

Fig. 5 Correlations between scWAT transcript levels and euglycaemichyperinsulinaemic clamp results. Principal component analysis was performed on the 73 transcripts downregulated after 12 weeks of exercise in men with dysglycaemia (see Fig. 3). The first principal component (PC1) correlated with GIR at (a) baseline and (b) after 12 weeks of exercise intervention, and (c) with change in GIR in response to 12 weeks of exercise intervention (principal component analysis was performed on change scores). (d) Transcript levels at baseline predicted the change in GIR in response to 12 weeks of exercise. Trend lines with standard error of estimates (SEEs; grey areas around the black regression lines) are presented. (e) The top ten most significant Spearman's correlations between transcript levels and GIR (see also ESM Table 1). The results were similar using either Spearman's or Pearson's correlations. $* * p<0.01$, $* * * p<0.001$ for the correlation between the gene and GIR

Pathway analysis of mRNA sequencing results Pathway analysis was performed using MSigDB (The Molecular Signatures Database; http://software.broadinstitute.org/gsea/ msigdb) KEGG pathways. Differentially expressed transcripts were tested for significant overlaps with these pathways using hypergeometric tests [39]. The BenjaminiHochberg procedure was used to correct $p$ values [40].
Upstream transcriptional regulators of the observed transcript changes were identified using Qiagen Upstream Regulator Analysis (Hilden, Germany).

\section{Secretory proteins}

We used the MetazSecKB (the metazoa [human \& animal] protein subcelluar location, secretome and subcellular proteome database; http://bioinformatics.ysu.edu/secretomes/ animal/index.php) knowledgebase to identify transcripts encoding secreted proteins. MetazSecKB identifies secretory proteins based on either curated evidence of secretion (annotated and reviewed in the UniProtKB/Swiss-Prot dataset) or computationally predicted secretory protein sequences, without containing transmembrane domains or endoplasmic reticulum retention signals, using several tools (SignalP4 [41], Phobius [42], TargetP [43] and WoLF PSORT [44]).

\section{scWAT cell type transcript markers}

We selected high-specificity markers of scWAT cell types (adipocytes, macrophages, leucocytes and progenitor cells) based on the study by Ehrlund et al [45], and markers of M1-like and M2-like macrophages from the study by Hill et al [46].

\section{Plasma analyses}

Plasma samples of secreted frizzled-related protein 4 (SFRP4) (catalogue no. SEF878Hu, Cloud-Clone, Houston, TX, USA), total and high-molecular-weight adiponectin (catalogue no. DRP300 and DHWAD0, R\&D Systems, Minneapolis, MN, USA), IL-6 (catalogue no. HS600B, R\&D Systems) and leptin (catalogue no. KAC2281, Invitrogen, Carlsbad, CA, USA) were measured in duplicate using ELISA according to the manufacturer's protocol. Absorbance was determined using a microplate reader (Titertec Multiscan Plus; EFLAB, Helsinki, Finland), which was set to 450 or $490 \mathrm{~nm}$ depending on the specific protocol. Standard curves for all proteins were generated using a best-fit curve.

\section{External datasets}

We compared our results with those from two other independent studies in obese, insulin-resistant participants by using the Array Express (EMBL-EBI), and the E-GEOD-70529 [47] and E-GEOD-26637 [48] datasets, respectively. The R packages Oligo [31] and LIMMA [49] were used for analysis.

\section{Statistics}

Data were modelled using parametric or non-parametric methods, as appropriate and specified along with each 
analysis. $p$ values were considered significant at $\alpha=0.05$. All data were analysed using R v3.3.3 [50]. We performed principal component analysis to enable the study of intercorrelated measures by producing linear combinations (principal components), where the first principal component is the linear sum of the measures that has the largest total variance [51].

\section{Results}

GIR increased by $45 \%$ and $38 \%$ in men with dysglycaemia and normoglycaemia, respectively (Table 1). Participant characteristics are presented in Table 1. At baseline, men with dysglycaemia had significantly more adipose tissue, lower $\dot{V} \mathrm{O}_{2 \max }$ and impaired glucose metabolism, and a non-significant tendency to higher plasma hsCRP levels $(p<0.06)$ vs control individuals. Body composition, physical fitness and glucose metabolism improved similarly for both groups in response to 12 weeks of exercise (Table 1), as reported previously [26].

\section{scWAT transcriptomics response to 12 weeks of exercise}

Intersected results from all three approaches to mRNA sequencing analyses revealed seven transcripts responding to 12 weeks of exercise among control participants (Fig. 2a,c), as compared with 90 transcripts in men with dysglycaemia (Fig. 2b,d). Three transcripts were upregulated and four transcripts were downregulated in control participants (Fig. 2a and Table 2), while 17 transcripts were upregulated and 73 transcripts were downregulated in men with dysglycaemia (Fig. $2 \mathrm{~b}$ and Table 3). Evidence for encoding secretory proteins existed for five of seven transcripts for control participants (Table 2) and for 62 of 90 transcripts for men with dysglycaemia (Table 3).

For the 73 transcripts that were downregulated in men with dysglycaemia, absolute levels of most mRNAs were higher in men with dysglycaemia as compared with control participants at baseline (Fig. 3). The differences in absolute transcript levels between the two groups were attenuated after 12 weeks of exercise (Fig. 3).

Our mRNA sequencing data were highly coherent across statistical approaches and across different technologies, including as RT-PCR (Table 4 and Fig. 4), but also cDNA microarrays from a subset of the samples (after 12 weeks of exercise, SFRP4 expression in control participants was reduced by $76.5 \%[p=0.13 ; n=4]$, whilst in men with dysglycaemia it was reduced by $55.3 \%[p<0.001 n=7])$.

\section{scWAT mRNA levels and insulin sensitivity}

We applied principal component analyses to the 73 downregulated transcripts in men with dysglycaemia after 12 weeks of exercise, and correlated the first principal component to insulin sensitivity, measured as GIR (Fig. 5). The first principal component correlated with GIR at baseline (Fig. 5a) and after the 12 week intervention (Fig. 5b). We also observed significant correlations between changes in GIR in response to the 12 week intervention and changes in the levels of these transcripts (Fig. 5c), and baseline transcript levels predicted GIR change in response to the intervention (Fig. 5d). The transcripts exhibiting the most significant Spearman's correlations with GIR are presented in Fig. 5e, and all transcript correlations with GIR are presented in the electronic supplementary material (ESM) Table 1.

\section{scWAT pathway and upstream regulator analyses}

Transcripts responding to 12 weeks of exercise in men with dysglycaemia (Table 3 ) overlapped with several immunerelated pathways, such as leucocytes, transendothelial migration, toll-like receptor signalling, and $\mathrm{B}$ and $\mathrm{T}$ cell receptor signalling (Fig. 6a). These transcripts (Table 3) also correlated with plasma hsCRP levels (ESM Table 2), and might form a part of a cytokine signalling network regulated by the transcription factors SRY-box 9 (SOX9), transcriptional and immune response regulator (TCIM) and RAR-related orphan receptor C, isoform CRA_a (RORC) (Fig. 6b).

\section{scWAT cell populations}

The 73 downregulated transcripts after 12 weeks of exercise in men with dysglycaemia were highly related to immune- and macrophage-related processes, based on gene-set enrichment analyses (Fig. 6a). We monitored the top 100 high-specificity transcript markers of human scWAT adipocytes, macrophages, leucocytes and progenitor cells, as according to Ehrlund et al [45]. Adipocyte-related transcript levels were lower and macrophage-related transcript levels were higher in men with dysglycaemia as compared with the control group (Fig. 6c,d). Macrophage- and leucocyte-related transcript levels decreased in men with dysglycaemia after 12 weeks of exercise intervention, whereas no changes were observed in the control participants (Fig. 6d,e). We also analysed high-specificity markers of human scWAT M1-like and M2-like macrophages, as according to Hill et al [46]. M2-like, but not M1-like, macrophage-related transcripts were more highly expressed in men with dysglycaemia as compared with control participants (Fig. 6g,h), and M2-like macrophage-related transcripts decreased in men with dysglycaemia after 12 weeks of the exercise intervention, whereas no change was observed in the control participants (Fig. 6h). M2-like macrophage transcript levels correlated negatively to GIR and plasma adiponectin levels (Fig. 6i) and scWAT ADIPOQ transcript levels (Fig. 6j) at baseline. However, the correlation between M2-like macrophage transcript levels and GIR disappeared 
a

Per cent enrichment

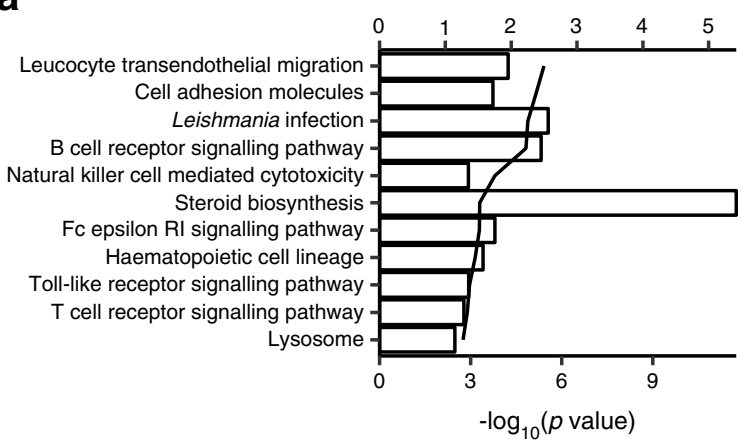

b

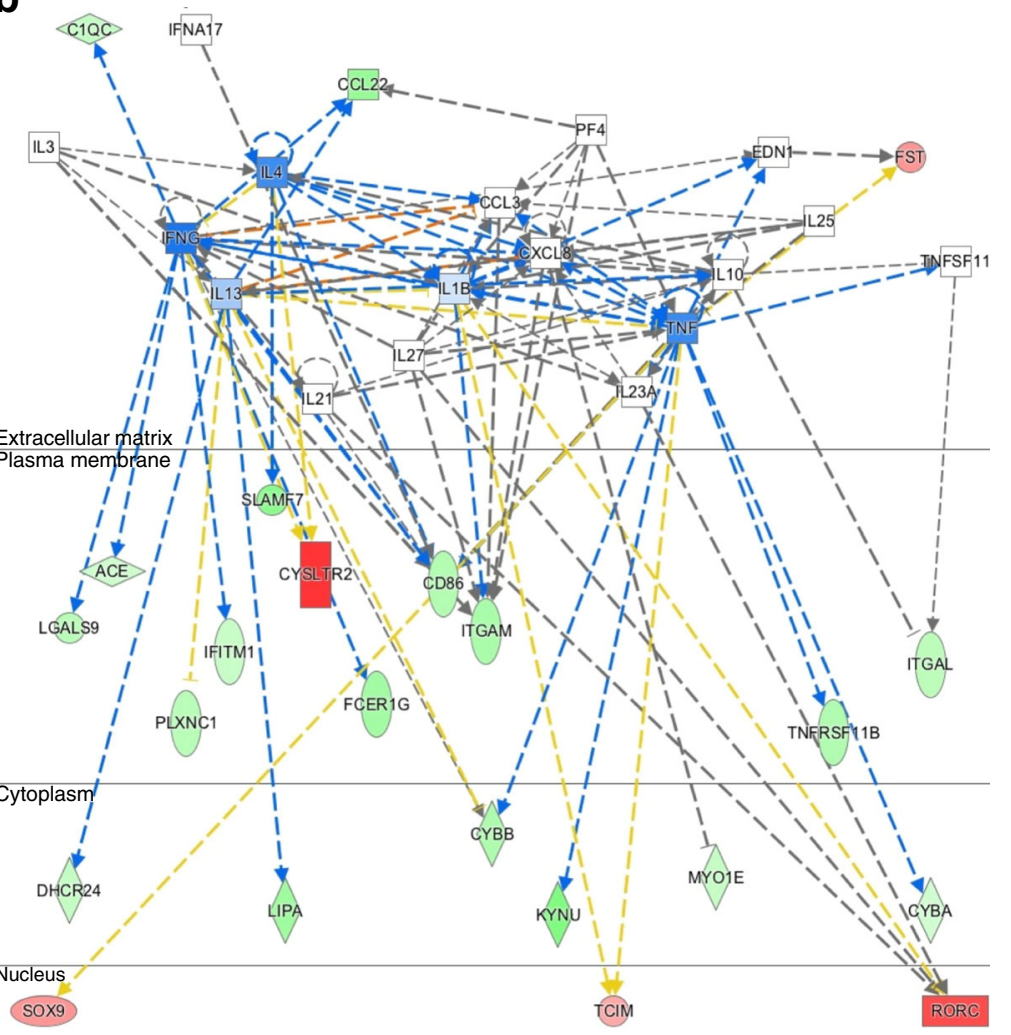

\begin{tabular}{|l|}
\hline High \\
Increased measurement \\
Decreased measurement \\
Predicted relationships \\
Leads to activation -------- \\
Leads to inhibition ------- \\
Inconsistent findings ------- \\
Effects not predicted -------
\end{tabular}

sox9
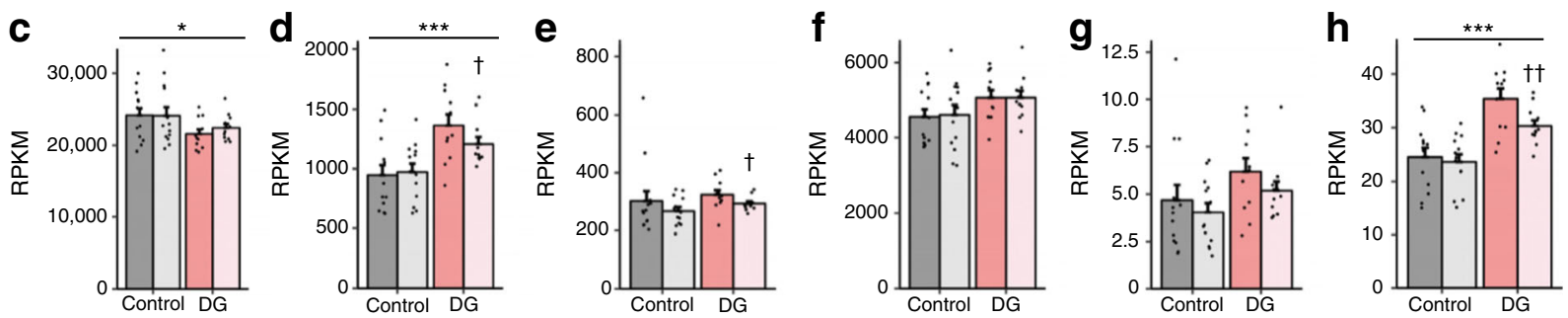

i

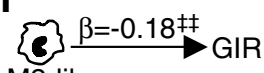

M2-like

j

[c] $\stackrel{\beta=-0.18 \text { 㧊 }}{\longrightarrow}$ GIR

ce $\stackrel{\beta=-1.44 \text { 势 }}{\longrightarrow}$ P-adiponectin

M2-like

M2-like

Ce-like $\stackrel{\beta=-10.5^{\text {护 }}}{\longrightarrow}$ ADIPOQ

P-adiponectin

[C] $\beta=-0.11$ GIR

M2-like

GIR

$$
\begin{aligned}
& \text { M2-like } \\
& \text { Mediated effect: } 39.5 \% \neq
\end{aligned}
$$


Fig. 6 Pathways, cell types and mediation analyses. (a) Pathwayenrichment analysis of scWAT transcripts regulated after 12 weeks of exercise in dysglycaemic men, and (b) upstream regulatory network analysis. (c-h) Analyses of main cell populations in scWAT: (c) adipocytes, (d) macrophages, (e) leucocytes, (f) progenitor cells, (g) M1-like macrophages and (h) M2-like macrophages. Bar plots represent means \pm SEM. ${ }^{*} p<0.05, * * * p<0.001$ for between-group comparison (dysglycaemia vs control participants) at baseline using unpaired $t$ tests. ${ }^{\dagger} p<0.05,{ }^{\dagger \dagger} p<0.01$ for within-group comparison (after [light grey/light red] vs before [dark grey/dark red] the 12 week intervention) using paired $t$ tests. (i, j) Mediation analyses between M2like macrophage transcript levels and (i) plasma (P) adiponectin levels or (j) scWAT ADIPOQ transcript levels, and GIR. ${ }^{\ddagger} p<0.05,{ }^{\star \star} p<0.01$. DG, participants with dysglycaemia; RPKM, reads per kilobase of transcript per million mapped reads

after adjusting for adiponectin levels (Fig. 6i,j). Group differences and responses to 12 weeks of exercise for all of the top 100 transcript markers for each scWAT cell type are presented in ESM Tables 3-6.

\section{Effects of weight loss and insulin}

To explore potential mechanisms behind the dysglycaemiaspecific effects of 12 weeks of exercise intervention on scWAT, we analysed the transcripts that changed in response to exercise in men with dysglycaemia only (Table 3 ). We investigated if the change in transcripts could be due to weight loss by comparing our exercise-changed transcripts to those changed in scWAT after weight loss in obese and insulinresistant participants in the study of Magkos et al [47] (Fig. 7a). We also evaluated if the change in these transcripts might be due to hyperinsulinaemia by studying data from human scWAT before and after $3 \mathrm{~h}$ of insulin infusion in obese and insulin-resistant participants compared with lean and insulinsensitive participants in the study by Soronen et al [48] (Fig. $7 \mathrm{~b}, \mathrm{c})$.

Thirteen transcripts were associated with genes changed in response to weight loss (Fig. 7a), and these exhibited the same direction of regulation as seen after 12 weeks of exercise intervention (Table 3). Eight transcripts in the study by Soronen et al [48] were associated with hyperinsulinaemia (Fig. 7b) and exhibited the opposite directions of regulation as seen after 12 weeks of exercise intervention (Table 3). Eight transcripts were differently regulated in response to insulin infusion between insulin-resistant and insulin-sensitive participants (Fig. 7c).

\section{scWAT mRNA levels and plasma adipokine concentrations}

$L E P$ and $A D I P O Q$ mRNA levels displayed significant correlations with plasma leptin and high-molecular-weight adiponectin concentrations in both groups of participants at baseline (Fig. 8). Similar results were also observed for
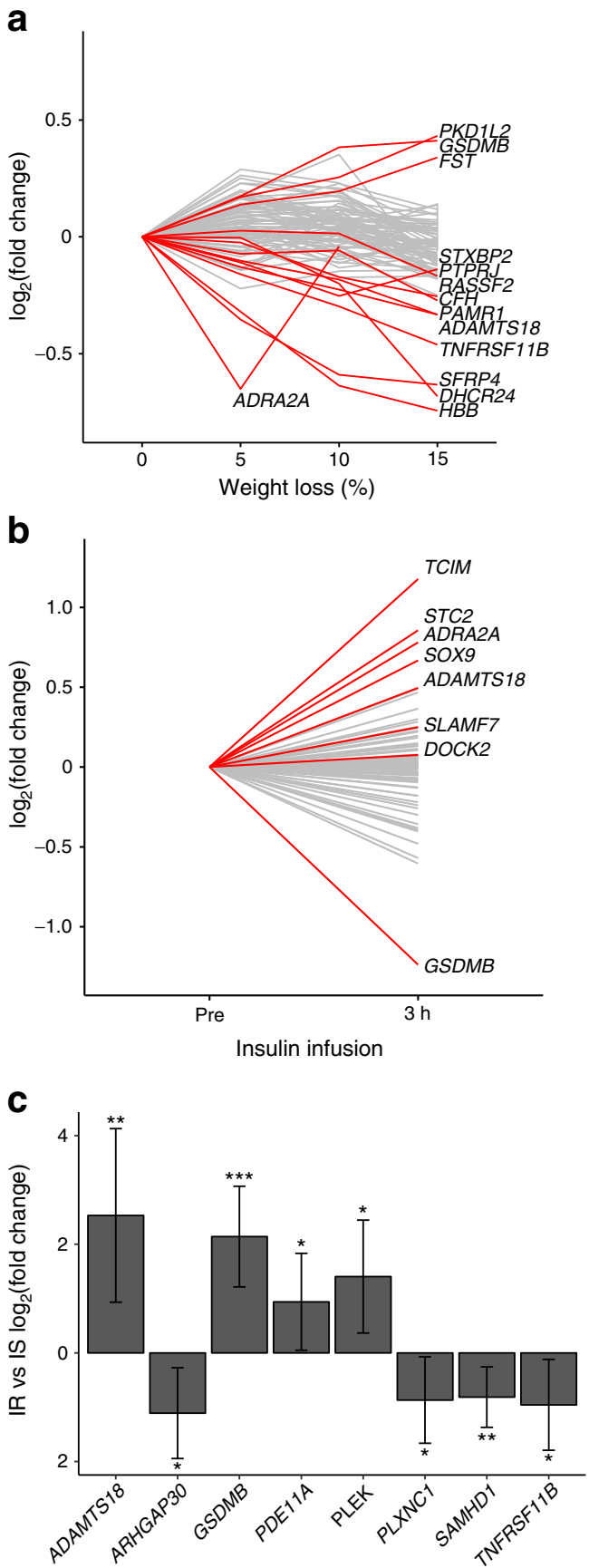

Fig. 7 Effects of weight loss and hyperinsulinaemia on subcutaneous adipose tissue transcript levels. (a) Effects of moderate and subsequent progressive weight loss from the study by Magkos et al [47]. Grey lines, $p>0.05$; red lines, $p<0.05$ ( 5,10 or $15 \%$ weight loss vs baseline). (b) Effects of $3 \mathrm{~h}$ of insulin infusion (grey lines, $p>0.05$; red lines, $p<0.05$ $3 \mathrm{~h}$ insulin infusion vs baseline), and (c) differences in response to insulin infusion between insulin-resistant (IR) and insulin-sensitive (IS) participants based on data from the study by Soronen et al [48]. The bar plot represents $\log _{2}$ (fold change) $\pm 95 \%$ CIs for the response to insulin infusion in insulin-resistant vs insulin-sensitive participants. $* p<0.05$, $* * p<0.01, * * * p<0.001$

IL- 6 and total adiponectin, and for values after 12 weeks and for changes observed during the intervention (data not shown). 


\section{Plasma adipokines response to exercise}

Because SFRP4 mRNA showed the largest change in response to the exercise intervention (i.e. lowest $p$ values) across all applied methods (Table 3), and has a known signalling peptide (Table 3), we investigated the effect of exercise on plasma SFRP4 levels. Whereas scWAT SFRP4 mRNA levels did not correlate to plasma SFRP4 concentrations in control participants (Fig. 9a), a significant correlation was seen in men with dysglycaemia (Fig. 9b). Furthermore, no change in plasma SFRP4 concentration was seen in the control group after 12 weeks of exercise (Fig. 9c), whereas a reduction was observed in men with dysglycaemia.

\section{Skeletal muscle mRNA levels and plasma adipokine levels}

No correlations were observed between skeletal muscle mRNA levels and plasma concentrations of leptin, total or high-molecular-weight adiponectin, IL-6 or SFRP4 (data not shown).
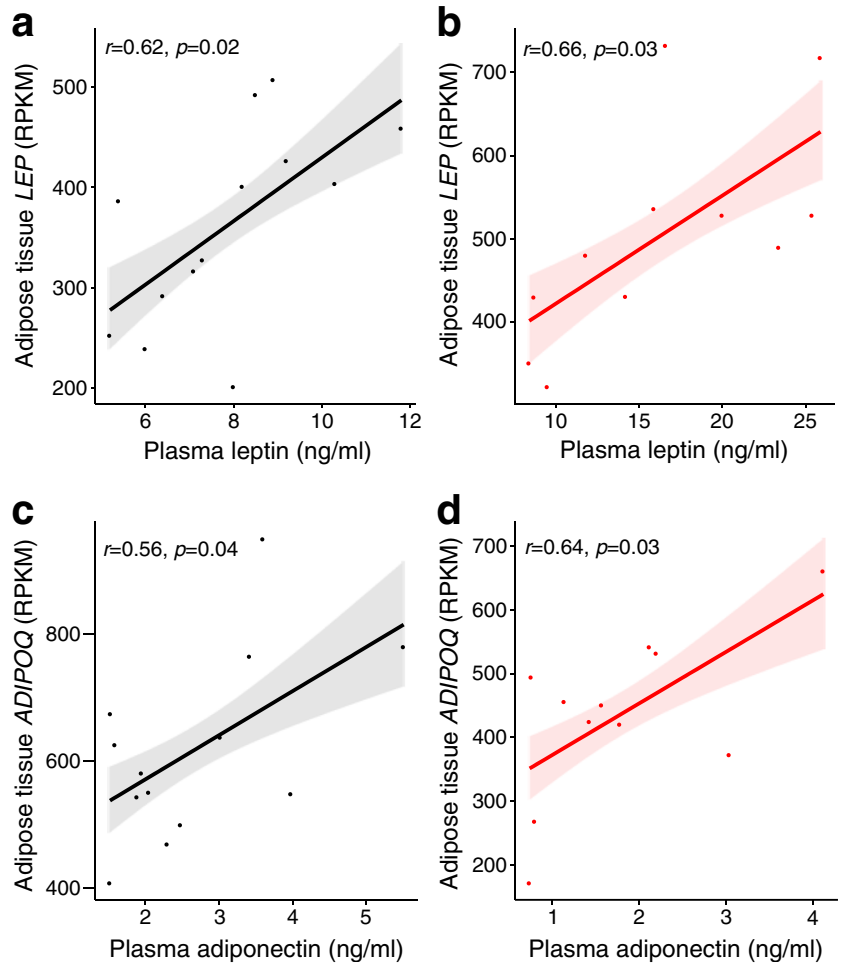

Fig. 8 Correlations between transcript levels in scWAT and protein concentrations in plasma. scWAT transcript levels (quantified by mRNA sequencing) correlated with corresponding plasma protein concentrations for $(\mathbf{a}, \mathbf{b})$ leptin and $(\mathbf{c}, \mathbf{d})$ high-molecular-weight adiponectin in both groups at baseline. The results were similar using either Spearman's or Pearson's correlations. Black lines and dots, control participants; red lines and dots, men with dysglycaemia. Trend lines with standard error of estimates (SEEs; shaded areas) are presented. RPKM, reads per kilobase per million mapped reads

\section{Discussion}

Our main findings were: (1) a substantial difference between change in scWAT transcript levels in response to 12 weeks of exercise among men with dysglycaemia as compared with the control group; (2) some dysglycaemia-specific transcripts were upregulated, but most transcripts were downregulated after 12 weeks of exercise, and were related to inflammation and macrophages; (3) absolute levels of these immune-related transcripts were elevated in men with dysglycaemia at baseline, but were normalised after 12 weeks of exercise, as compared with control participants; (4) levels of these immunerelated transcripts correlated negatively with insulin sensitivity in several comparisons; and (5) exercise responses in the scWAT secretome may be reflected in altered plasma concentration of adipokines such as SFRP4.

Whereas several scientists have described adipose tissue responses to different weight-loss regimens in a variety of human populations and animal models [15, 24, 35, 52], there
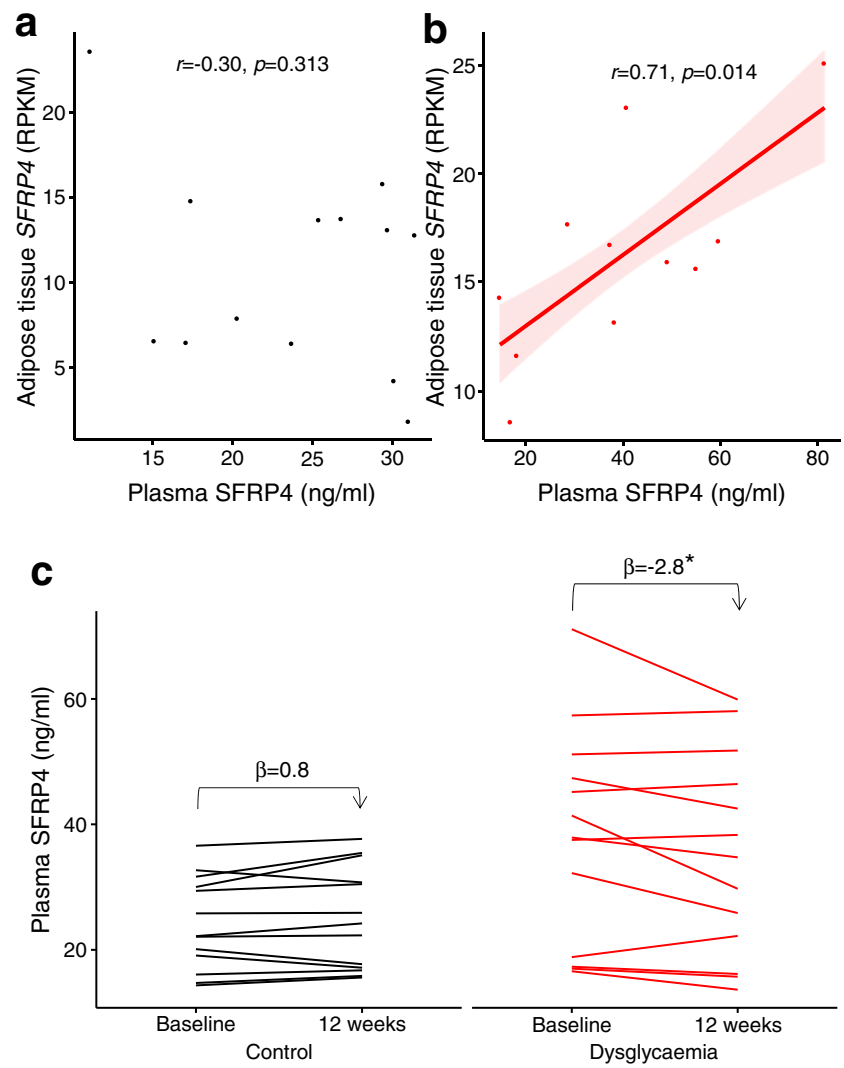

Fig. 9 Analysis of SFRP4. (a) Plasma SFRP4 concentrations did not correlate with SFRP4 levels in scWAT in men with normoglycaemia (control), but did correlate in (b) men with dysglycaemia. The results were similar using either Spearman's and Pearson's correlations. Trend lines with standard error of estimates (SEEs; shaded areas) are presented. (c) Plasma SFRP4 concentrations did not change after 12 weeks of exercise in men with normoglycaemia, but decreased in men with dysglycaemia. Black lines and dots, control participants; red lines and dots, men with dysglycaemia. RPKM, reads per kilobase of transcript per million mapped reads. ${ }^{*} p<0.05$, paired $t$ tests were performed 
is a lack of studies on the response to exercise, and especially in reporting results after a long-term period of exercise in humans $[15,20,21]$. One study applied microarrays on scWAT before and after 6 months of an exercise intervention in 14 postmenopausal women and found no apparent alterations in transcript levels [24]. Another study among 25 obese, premenopausal women revealed no changes in the mRNA levels of $L E P, A D I P O Q, I L 6$ and TNF after 12 weeks of bicycle ergometer training [22]. Although large differences exist between these studies and ours with respect to exercise protocols, the sex, age, BMI and ethnicity of the participants and the applied technologies, the most important difference may be that the women in these earlier studies were normoglycaemic and insulin sensitive. Hence, the results were in line with those seen among our normoglycaemic and insulin-sensitive control group.

One striking observation in our data is the effect of long-term exercise on reducing levels of inflammatory mRNAs in scWAT among the overweight, dysglycaemic participants. Our pathway analysis and assessment of high-specificity transcript markers of scWAT cell populations [45] indicated less immune cell infiltration, especially of leucocytes and macrophages, after 12 weeks of the exercise intervention in men with dysglycaemia. This is in line with previous studies suggesting that exercise may reduce immune cell infiltration in the stromal vascular fraction of adipose tissue and positively influence adipose tissue macrophage phenotypes [17, 18, 23]. However, these studies focused on visceral adipose tissue [17, 18, 23], not scWAT.

It should be noted that the different transcriptional alterations in scWAT between the two groups in our study occurred without significant changes in diet, BMI, plasma fasting insulin or C-peptide (Table 1). There were also similar reductions in the amount of scWAT and visceral adipose tissue [26] and similar increases in GIR and $\dot{V} \mathrm{O}_{2 \max }$ between the two groups (Table 1). Thus, we can only speculate as to why exercise responses in scWAT differed between the two groups, but we suggest that it might relate to differences in fat tissue at baseline (Table 1). Our men with dysglycaemia had more scWAT (Table 1) and increased scWAT inflammation-related transcript levels (Figs. 3 and 6) compared with the control group at baseline. Whereas reduced amounts of scWAT after 12 weeks of exercise in men with dysglycaemia might counteract inflammation-responses associated with expanded adipose tissue $[5,6]$, no such alterations occurred in normal-weight men, perhaps because their scWAT only changed within a physiological range. We analysed scWAT transcript responses to weight loss (mostly fat mass) and hyperinsulinaemia (insulin infusion for $3 \mathrm{~h}$ ) in obese and insulin-resistant humans [47, 48]. These analyses demonstrated that some transcripts responding to 12 weeks of exercise intervention in men with dysglycaemia also responded to fat loss or hyperinsulinaemia. Thus, for these transcripts, the observed responses in our data might not be due to exercise per se, but rather due to loss of fat mass and/or improved insulin sensitivity. However, for the majority of transcripts (Table 3), there may be a direct effect of long-term exercise and alleviation of insulin resistance in men with dysglycaemia.

Although human data are generally lacking regarding scWAT and exercise, reports from experiments in rodents have demonstrated profound effects of exercise on scWAT [4]. Transplanting scWAT from mice performing 11 days of cage wheel exercise into sedentary mice dramatically improved glucose tolerance [4]. This effect lasted for 9 days and was related to increased skeletal muscle and intrascapular brown adipose tissue glucose uptake, perhaps related to alterations in more than 250 putative adipokines, possible mediating the effect on glucose tolerance [4]. Although the relevance of these findings to humans remains uncertain, they are in line with our results showing that especially macrophage-related transcript expression seems to be negatively correlated with insulin sensitivity, and that much of this effect may relate to interplay with adipocytes through, for example, adiponectin (Fig. 6 and ESM Tables 1-6) [53]. Thus, both data from Stanford et al [4] and our study suggest that some of the effects of long-term exercise on insulin sensitivity are mediated by alterations in scWAT.

Previous studies on exercise and adipokines have described minor effects, at least compared with different weight-loss regimes [20]. These observations are in agreement with our study, which revealed modest changes in mRNA levels (Fig. 2) and minor changes in plasma adipokine concentrations (Fig. 9). However, subtle effects might still reflect important adaptations; for example, SFRP4 has been shown to be secreted from human scWAT explants [54], and scWAT is the major contributor to plasma SFRP4 concentrations, correlating with indices of obesity and insulin resistance [55]. A mechanism linking elevated plasma SFRP4 concentration to type 2 diabetes involves inflammation in pancreatic islets and reduced insulin secretion [56].

The main limitation of our study is the small sample size of white men only, providing low statistical power and reduced generalisability. However, we applied state-of-the art technologies on human participants who were overweight and of normal weight, and with and without dysglycaemia, who underwent a strictly controlled high-intensity exercise intervention, and compared several statistical approaches across multiple technological platforms to minimise bias. Moreover, the published literature on adipokines and exercise is limited and incomplete, especially in regard to scWAT.

\section{Conclusion}

We discovered a pronounced effect of long-term exercise on scWAT in overweight men with dysglycaemia, whereas small alterations were observed in normal-weight men with 
normoglycaemia. The effect included normalisation of macrophage-related transcript levels, and was closely related to improved insulin sensitivity. The alterations in scWAT involved several secreted factors, and may be mirrored in alterations of plasma adipokine concentrations, at least for SFRP4. These findings indicate that scWAT may be an important mediator of exercise-induced improvements in insulin sensitivity for individuals at risk of developing type 2 diabetes.

Acknowledgements We thank A. Halsne, G. Vinje, K. E. Jahnsen, A. Heck, B. Nellemann (Department of Endocrinology, Oslo University Hospital), A. R. Enget (Department of Nutrition, University of Oslo), T. I. Gloppen, T. Dalen, H. Moen, M. A. Dahl, G. Grøthe, K. A Krog, Ø. Skattebo, E. Johansen, D. S. Tangen, K. K. Jensen, H. K. Stadheim, E. N. Rise (Norwegian School of Sport Sciences) and the Norwegian Sequencing Centre.

Data availability The data are available on request from the authors.

Funding This work was supported by grants from the Institute of Basic Medical Sciences, UiO, the Johan Throne-Holst Foundation for Nutrition Research, the Freia Medical Research Foundation, the 'Functional Genomics' and 'Infrastructure' programmes of the Research Council of Norway, the EU-financed FP7 project (NutriTech grant agreement no.: 289511) and the South-Eastern Regional Health Authorities.

Duality of interest The authors declare that there is no duality of interest associated with this manuscript.

Contribution statement SL analysed and prepared the data, and wrote the first draft of the manuscript. All authors interpreted the data and reviewed, revised and approved the manuscript. KIB and CAD initiated, designed and supervised the study. CAD is the guarantor of this work.

\section{References}

1. Knights AJ, Funnell AP, Pearson RC, Crossley M, Bell-Anderson KS (2014) Adipokines and insulin action: A sensitive issue. Adipocyte 3(2):88-96. https://doi.org/10.4161/adip.27552

2. Drevon CA (2005) Fatty acids and expression of adipokines. Biochim Biophys Acta 1740(2):287-292. https://doi.org/10.1016/ j.bbadis.2004.11.019

3. Görgens SW, Eckardt K, Jensen J, Drevon CA, Eckel J (2015) Exercise and regulation of adipokine and myokine production. In: Claude B (ed) Progress in molecular biology and translational science. Academic Press, Cambridge, pp 313-336

4. Stanford KI, Middelbeek RJ, Goodyear LJ (2015) Exercise effects on white adipose tissue: beiging and metabolic adaptations. diabetes 64(7):2361-2368. https://doi.org/10.2337/db15-0227

5. Boutens L, Stienstra R (2016) Adipose tissue macrophages: going off track during obesity. Diabetologia 59(5):879-894. https://doi. org/10.1007/s00125-016-3904-9

6. McLaughlin T, Deng A, Yee G et al (2010) Inflammation in subcutaneous adipose tissue: relationship to adipose cell size. Diabetologia 53(2):369-377. https://doi.org/10.1007/s00125-0091496-3

7. Bergmann K, Sypniewska G (2013) Diabetes as a complication of adipose tissue dysfunction. Is there a role for potential new biomarkers? Clin Chem Lab Med 51:177-185

8. Dolinkova M, Dostalova I, Lacinova Z et al (2008) The endocrine profile of subcutaneous and visceral adipose tissue of obese patients. Mol Cell Endocrinol 291(1-2):63-70. https://doi.org/10. 1016/j.mce.2008.05.001

9. Huber J, Kiefer FW, Zeyda M et al (2008) CC chemokine and CC chemokine receptor profiles in visceral and subcutaneous adipose tissue are altered in human obesity. J Clin Endocrinol Metab 93(8): 3215-3221. https://doi.org/10.1210/jc.2007-2630

10. Samaras K, Botelho NK, Chisholm DJ, Lord RV (2010) Subcutaneous and visceral adipose tissue gene expression of serum adipokines that predict type 2 diabetes. Obesity (Silver Spring) 18(5):884-889. https://doi.org/10.1038/oby.2009.443

11. Anderssen S, Holme I, Urdal P, Hjermann I (1995) Diet and exercise intervention have favourable effects on blood pressure in mild hypertensives: the Oslo Diet and Exercise Study (ODES). Blood Press 4(6):343-349. https://doi.org/10.3109/08037059509077619

12. Anderssen SA, Hjermann I, Urdal P, Torjesen PA, Holme I (1996) Improved carbohydrate metabolism after physical training and dietary intervention in individuals with the "atherothrombogenic syndrome". Oslo Diet and Exercise Study (ODES). A randomized trial. J Intern Med 240(4):203-209. https://doi.org/10.1046/j.1365-2796. 1996.22848000.x

13. Torjesen PA, Birkeland KI, Anderssen SA, Hjermann I, Holme I, Urdal P (1997) Lifestyle changes may reverse development of the insulin resistance syndrome. The Oslo Diet and Exercise Study: a randomized trial. Diabetes Care 20(1):26-31. https://doi.org/10. 2337/diacare.20.1.26

14. Anderssen SA, Holme I, Urdal P, Hjermann I (1998) Associations between central obesity and indexes of hemostatic, carbohydrate and lipid metabolism. Results of a 1-year intervention from the Oslo Diet and Exercise Study. Scand J Med Sci Sports 8(2):109 115

15. Thompson D, Karpe F, Lafontan M, Frayn K (2012) Physical activity and exercise in the regulation of human adipose tissue physiology. Physiol Rev 92(1):157-191. https://doi.org/10.1152/ physrev.00012.2011

16. Stanford KI, Lynes MD, Takahashi H et al (2018) 12,13-diHOME: an exercise-induced lipokine that increases skeletal muscle fatty acid uptake. Cell Metab 27(5):1111-1120.e1113. https://doi.org/ 10.1016/j.cmet.2018.03.020

17. Karsten K, Frank CM, Klaus E, Robert R (2014) Immune and inflammatory signaling pathways in exercise and obesity. Am J Lifestyle Med 10:268-279

18. Goh J, Goh KP, Abbasi A (2016) Exercise and adipose tissue macrophages: new frontiers in obesity research? Front Endocrinol 7:65

19. Sakurai T, Ogasawara J, Shirato K et al (2017) Exercise training attenuates the dysregulated expression of adipokines and oxidative stress in white adipose tissue. Oxidative Med Cell Longev 2017: 9410954

20. Campbell KL, Landells CE, Fan J, Brenner DR (2017) A systematic review of the effect of lifestyle interventions on adipose tissue gene expression: implications for carcinogenesis. Obesity (Silver Spring) 25(Suppl 2):S40-S51. https://doi.org/10.1002/oby.22010

21. Van Pelt DW, Guth LM, Horowitz JF (2017) Aerobic exercise elevates markers of angiogenesis and macrophage IL- 6 gene expression in the subcutaneous adipose tissue of overweight-to-obese adults. J Appl Physiol 123(5):1150-1159. https://doi.org/10.1152/ japplphysiol.00614.2017

22. Polak J, Klimcakova E, Moro C et al (2006) Effect of aerobic training on plasma levels and subcutaneous abdominal adipose tissue gene expression of adiponectin, leptin, interleukin 6, and tumor necrosis factor alpha in obese women. Metab Clin Exp 55(10): 1375-1381. https://doi.org/10.1016/j.metabol.2006.06.008

23. Catenacci VA, Wyatt HR (2007) The role of physical activity in producing and maintaining weight loss. Nat Clin Pract Endocrinol Metab 3(7):518-529. https://doi.org/10.1038/ncpendmet0554

24. Campbell KL, Foster-Schubert KE, Makar KW et al (2013) Gene expression changes in adipose tissue with diet- and/or exercise- 
induced weight loss. Cancer Prev Res 6(3):217-231. https://doi. org/10.1158/1940-6207.CAPR-12-0212

25. Pourteymour S, Eckardt K, Holen T et al (2017) Global mRNA sequencing of human skeletal muscle: search for novel exerciseregulated myokines. Molecular metabolism 6(4):352-365. https:// doi.org/10.1016/j.molmet.2017.01.007

26. Langleite TM, Jensen J, Norheim F et al (2016) Insulin sensitivity, body composition and adipose depots following $12 \mathrm{w}$ combined endurance and strength training in dysglycemic and normoglycemic sedentary men. Arch Physiol Biochem 122(4): 167-179. https://doi.org/10.1080/13813455.2016.1202985

27. Andersen LF, Nes M, Lillegaard IT, Sandstad B, Bjorneboe GE, Drevon CA (1995) Evaluation of a quantitative food frequency questionnaire used in a group of Norwegian adolescents. Eur J Clin Nutr 49(8):543-554

28. Andersen LF, Solvoll K, Johansson LR, Salminen I, Aro A, Drevon CA (1999) Evaluation of a food frequency questionnaire with weighed records, fatty acids, and alpha-tocopherol in adipose tissue and serum. Am J Epidemiol 150(1):75-87. https://doi.org/10.1093/ oxfordjournals.aje.a009921

29. DeFronzo RA, Tobin JD, Andres R (1979) Glucose clamp technique: a method for quantifying insulin secretion and resistance. Am J Phys 237:E214-E223

30. Stefan D, Cesare FD, Andrasescu A et al (2009) Quantitation of magnetic resonance spectroscopy signals: the jMRUI software package. Meas Sci Technol 20(10):104035. https://doi.org/10. 1088/0957-0233/20/10/104035

31. Carvalho BS, Irizarry RA (2010) A framework for oligonucleotide microarray preprocessing. Bioinformatics 26(19):2363-2367. https://doi.org/10.1093/bioinformatics/btq431

32. Trapnell C, Roberts A, Goff L et al (2012) Differential gene and transcript expression analysis of RNA-seq experiments with TopHat and Cufflinks. Nat Protoc 7(3):562-578. https://doi.org/ 10.1038/nprot.2012.016

33. Li H, Handsaker B, Wysoker A et al (2009) The sequence alignment/map format and SAMtools. Bioinformatics 25(16): 2078-2079. https://doi.org/10.1093/bioinformatics/btp352

34. Langmead B, Salzberg SL (2012) Fast gapped-read alignment with Bowtie 2. Nat Methods 9(4):357-359. https://doi.org/10.1038/ nmeth.1923

35. Quinlan AR, Hall IM (2010) BEDTools: a flexible suite of utilities for comparing genomic features. Bioinformatics 26(6):841-842. https://doi.org/10.1093/bioinformatics/btq033

36. Pyl PT, Anders S, Huber W (2014) HTSeq - a Python framework to work with high-throughput sequencing data. Bioinformatics 31 : 166-169

37. Robinson MD, McCarthy DJ, Smyth GK (2010) edgeR: a Bioconductor package for differential expression analysis of digital gene expression data. Bioinformatics 26(1):139-140. https://doi. org/10.1093/bioinformatics/btp616

38. Anders S, Huber W (2010) Differential expression analysis for sequence count data. Genome Biol 11(10):R106. https://doi.org/ 10.1186/gb-2010-11-10-r106

39. Subramanian A, Kuehn H, Gould J, Tamayo P, Mesirov JP (2007) GSEA-P: a desktop application for Gene Set Enrichment Analysis. Bioinformatics 23(23):3251-3253. https://doi.org/10.1093/ bioinformatics/btm369

40. Benjamini Y (2010) Discovering the false discovery rate. J R Stat Soc Ser B Stat Methodol 72(4):405-416. https://doi.org/10.1111/j. 1467-9868.2010.00746.x
41. Petersen TN, Brunak S, von Heijne G, Nielsen H (2011) SignalP 4.0: discriminating signal peptides from transmembrane regions. Nat Methods 8(10):785-786. https://doi.org/10.1038/nmeth.1701

42. Käll L, Krogh A, Sonnhammer ELL (2007) Advantages of combined transmembrane topology and signal peptide prediction-the Phobius web server. Nucleic Acids Res 35:W429-W432. https:/ doi.org/10.1093/nar/gkm256

43. Emanuelsson O, Nielsen H, Brunak S, von Heijne G (2000) Predicting subcellular localization of proteins based on their Nterminal amino acid sequence. J Mol Biol 300(4):1005-1016. https://doi.org/10.1006/jmbi.2000.3903

44. Horton P, Park K-J, Obayashi T et al (2007) WoLF PSORT: protein localization predictor. Nucleic Acids Res 35:W585-W587. https:// doi.org/10.1093/nar/gkm259

45. Ehrlund A, Acosta JR, Bjork C et al (2017) The cell-type specific transcriptome in human adipose tissue and influence of obesity on adipocyte progenitors. Scientific data 4:170164. https://doi.org/10. 1038/sdata.2017.164

46. Hill AA, Reid Bolus W, Hasty AH (2014) A decade of progress in adipose tissue macrophage biology. Immunol Rev 262(1):134-152. https://doi.org/10.1111/imr.12216

47. Magkos F, Fraterrigo G, Yoshino J et al (2016) Effects of moderate and subsequent progressive weight loss on metabolic function and adipose tissue biology in humans with obesity. Cell Metab 23(4): 591-601. https://doi.org/10.1016/j.cmet.2016.02.005

48. Soronen J, Laurila PP, Naukkarinen J et al (2012) Adipose tissue gene expression analysis reveals changes in inflammatory, mitochondrial respiratory and lipid metabolic pathways in obese insulin-resistant subjects. BMC Med Genet 5(1):9. https://doi.org/ 10.1186/1755-8794-5-9

49. Ritchie ME, Smyth GK, Phipson B et al (2015) limma powers differential expression analyses for RNA-sequencing and microarray studies. Nucleic Acids Res 43(7):e47-e47. https://doi.org/10. 1093/nar/gkv007

50. Team RC (2018) R: a language and environment for statistical computing. R Foundation for Statistical Computing, Vienna

51. Hillier TA, Rousseau A, Lange C et al (2006) Practical way to assess metabolic syndrome using a continuous score obtained from principal components analysis. Diabetologia 49(7):1528-1535. https://doi.org/10.1007/s00125-006-0266-8

52. Mathur SK, Jain P, Mathur P (2011) Microarray evidences the role of pathologic adipose tissue in insulin resistance and their clinical implications. J Obes 2011:16

53. Luo Y, Liu M (2016) Adiponectin: a versatile player of innate immunity. J Mol Cell Biol 8(2):120-128. https://doi.org/10.1093/ jmcb/mjw012

54. Ehrlund A, Mejhert N, Lorente-Cebrian S et al (2013) Characterization of the Wnt inhibitors secreted frizzled-related proteins (SFRPs) in human adipose tissue. J Clin Endocrinol Metab 98(3):E503-E508. https://doi.org/10.1210/jc.2012-3416

55. Garufi G, Seyhan AA, Pasarica M (2015) Elevated secreted frizzled-related protein 4 in obesity: a potential role in adipose tissue dysfunction. Obesity (Silver Spring) 23(1):24-27. https:// doi.org/10.1002/oby.20915

56. Mahdi T, Hanzelmann S, Salehi A et al (2012) Secreted frizzledrelated protein 4 reduces insulin secretion and is overexpressed in type 2 diabetes. Cell Metab 16(5):625-633. https://doi.org/10.1016/ j.cmet.2012.10.009

Publisher's note Springer Nature remains neutral with regard to jurisdictional claims in published maps and institutional affiliations. 